\title{
Patient-specific computational modeling of Cortical Spreading Depression via Diffusion Tensor Imaging
}

\author{
Julia M. Kroos ${ }^{1, *}$, Isabella Marinelli ${ }^{1}$, Ibai Diez ${ }^{2}$, Jesus M. Cortes ${ }^{2}$, \\ Sebastiano Stramaglia ${ }^{1,3}$, and Luca Gerardo-Giorda ${ }^{1}$ \\ ${ }^{1}$ Basque Center for Applied Mathematics, Bilbao, Spain \\ ${ }^{2}$ Computational Neuroimaging Lab, Biocruces Health Research \\ Institute, Cruces University Hospital, Barakaldo, Spain \\ ${ }^{3}$ Center of Innovative Technologies for Signal Detection and \\ Processing TIRES, Dipartimento di Fisica, Universita di Bari, Italy \\ * Basque Center for Applied Mathematics, Alameda de Mazarredo \\ 14, 48009 Bilbao, Bizkaia, Spain, jkroos@bcamath.org
}

February 24, 2017

\begin{abstract}
Cortical Spreading Depression (CSD), a depolarization wave originating in the visual cortex and traveling towards the frontal lobe, is commonly accepted as a correlate of migraine visual aura. As of today, little is known about the mechanisms that can trigger or stop such phenomenon. However, the complex and highly individual characteristics of the brain cortex suggest that the geometry might have a significant impact in supporting or contrasting the propagation of CSD. Accurate patient-specific computational models are fundamental to cope with the high variability in cortical geometries among individuals, but also with the conduction anisotropy induced in a given cortex by the complex neuronal organisation in the grey matter. In this paper we integrate a distributed model for extracellular potassium concentration with patient-specific diffusivity tensors derived locally from Diffusion Tensor Imaging data.
\end{abstract}

Keywords: Cortical Spreading Depression, Diffusion Tensor Imaging, patientspecific cortical geometry, finite element simulation, reaction-diffusion systems

\section{Introduction}

The symptoms of migraine - a prevailing disease for today's population - vary from unilateral severe headache and nausea to photophobia. The visual aura, 
affecting more than $30 \%$ of the migraine patients, has been deemed a neurophysiological correlate of the Cortical Spreading Depression (CSD) [1]. CSD is a wave of intense depolarisation that, originating in the visual cortex, propagates to the peripheral areas in approximately 20 minutes, causes a drastic failure of brain homeostasis, and is followed by a wave of depression of neuronal activity 2]. Although the mechanisms underlaying CSD are yet not fully understood, it is clear that wave propagation does depend on the medium characteristics [3] at large: besides physiological properties, fissures and sulci of the cortex influence the propagation of depolarisation waves and could stop the migraine in different positions depending on the patient.

Several mathematical tools, from microscopic, biophysical models accounting for various physiological quantities to phenomenological models usually defined through reaction diffusion equations, have been proposed in the past to model CSD. For a general overview of those models we refer the reader to [4, 5, 6, 7]. Biophysical models provide insights on interactions and dynamics at a cellular level, while phenomenological models are more suited for analyzing the general CSD propagation. As an example, Tuckwell and Miura proposed in [8] a model for the propagation of a spreading depression wave in a $1 \mathrm{D}$ heterogenous space: their model, by including potassium and calcium fluxes, extracellular diffusion and active transport pumps in a Hodgkin-Huxley like system of equations, is able to reproduce the basic qualitative properties of the spreading depression waves and to account for the annihilation of two colliding waves. In [9], Reggia and Montgomery use a reaction diffusion equation to describe the potassium changes in a simplified 2D array representation of the cortex, where they couple synaptic connectivity and extracellular potassium uptake at a single cell level. They project the simulated cortical activity onto the visual field to mimic the corresponding visual pattern: the potassium wave triggers irregular patches of highly activated areas on the visual field, supporting the theory of CSD underlying migraine aura. Recently, Mori proposed a model of electrodiffusion and osmotic water flow, to study tissue-level physiological phenomena such as CSD [10. Dahlem and Isle [11] explore the statistical properties of transient cortical wave patterns with a canonical reaction-diffusion model for the membrane potential, coupled with mean field inhibition.

Four hypothesis to explain the CSD propagation on the cortex have been proposed, and accepted at different levels. The potassium and glutamate hypotheses state that CSD propagates due to diffusion of potassium or glutamate in the extracellular space. The neuronal gap junction hypothesis suggests that CSD propagates through the opening of neuronal gap junctions. The glial hypothesis states that CSD is transmitted through glial gap junctions. The most accepted assumption is that of the extracellular potassium and glutamate diffusion, which is presumed to follow ordinary diffusion laws [7. In this paper, we follow the potassium assumption and we model the propagation of CSD by tracking the extracellular potassium.

Cardiac electrophysiological studies have long been dealing with patientspecific geometries (see, for instance, [12, 13, 14, 15, 16, 17, 18, and references therein), but much less has been done so far in the case of CSD, despite the fact 
that the far more complex, highly individual, characteristics of the brain cortex suggest that the geometry might have a significant impact in supporting or contrasting the propagation of the depolarization wave. More recent works on CSD started to focus on patient-specific simulations to optimise the personalised treatment: Dahlem and his collaborators, by means of the Gaussian curvature of the cortex (computable from MRI data), analyse local structures to target neuromodulation [19]. A method to simulate the propagation of CSD wavefronts on realistic geometries, based on representing neurons as current dipoles normal to the brain surface, was proposed in [20]. Some of the authors of this paper, by using a computational neural firing rate model distributed throughout a realistic cortical mesh, proposed in 21] a more global approach to study the propagation across the whole cortex, that allowed to identify brain regions featuring a peculiar behaviour during CSD propagation. In particular, some regions appeared to trap the propagating depolarization waves for longer times, and are likely to play a crucial role in the CSD propagation (and, possibly, to be able to stop it).

The approach introduced in 21] provides some important insights on the propagation on a realistic geometry. However, the interpretation of its state variable might appear somehow questionable from the physiological standpoint. In addition, it uses a constant, isotropic diffusion tensor for the whole cortical geometry. The complex structure of the neural connections in the grey matter make such isotropy assumption quite unrealistic. In this paper, we overcome such limitations by using a distributed model for the extracellular potassium dynamics, in which we integrate more personalized diffusion coefficients derived from Diffusion Tensor Imaging (DTI) data.

DTI is an imaging technique based on Magnetic Resonance Imaging (MRI) that measures, in every voxel of an MRI image, the diffusion of water molecules in different directions. The underlying idea is that random movements of water molecules are influenced and governed by its surrounding tissue structure and geometric organisation. Evidence that the water diffusion tensors obtained from DTI measurements can be a good estimation for the electrical conductivity tensor of different tissues is presented in [22, 23, 24]. In literature, DTI is principally used to asses water molecules diffusion in white matter tracts (see, for instance, [25]), which are characterized by strong anisotropies. Since also grey matter features morphological anisotropy (although at smaller degree with respect to white matter), we assume that DTI measurements on the cortex might account for diffusivity properties as well. Nevertheless, DTI data cannot be used just as they are: DTI data provide information on three-dimensional diffusion, while a cortical geometry reconstructed from MRI is a two-dimensional surface embedded in three-dimensional space. Thus, in order to integrate DTI information in the distributed model, 2D tensors have to be derived from the original 3D data. Since the computational domain is a triangulation of the reconstructed surface, and the numerical approximation of the distributed model is based on finite elements, these $2 \mathrm{D}$ tensors are derived locally on each element of the triangulation.

The paper is organized as follows. In section 2 we introduce the distributed 
extracellular potassium concentration model and discuss its variational formulation. In section 3 we describe the computational domain and the numerical approximation of the distributed model. Section 4 is devoted to the derivation of the surface diffusion tensors from the three-dimensional DTI data. In section 5 we present some numerical simulations, highlighting the differences with respect to using an isotropic conductivity tensor.

\section{A distributed model of Cortical Spreading De- pression}

A key property of neural cells is to produce an action potential (AP). It consists in a sudden variation in the transmembrane potential, called spike, followed by a recovering of the resting condition through a refractory period, during which the cell cannot be excited. In agreement with previous computational studies [26], we consider neurons at rest to have a background firing rate of $4 \mathrm{~Hz}$ while excited neurons fire with an average frequency of $64 \mathrm{~Hz}$

A detailed model for action potential, such as the ones introduced in [27, 28], would incur unnecessary high computational costs to reproduce a firing rate of $64 \mathrm{~Hz}$ during 20 minutes: representing such frequency would prompt the use of a time step in the order of $\Delta t=0.1 \mathrm{msec}$, resulting in around $12 \mathrm{M}$ time steps to be solved for simulating the CSD propagation during such a long time. In addition, the large amount of neurons in the grey matter would require an extremely detailed geometrical mesh to take into account every single one of them. To cope with this issue, we introduce here a slow variables model to describe the CSD-induced disruption, by tracking one correlate of the depolarization wave [6, 7. Owing to the potassium assumption for CSD, the state variable $u(x, t)$ represents here the extracellular potassium concentration at location $x$ and time $t$ (in seconds). Such phenomenological model for the extracellular potassium allows us to use a computational grid whose characteristic size is of the order of millimeters, the standard resolution of common MRI scanners. The model is a modification of the Roger-McCulloch variant [29] of the Fitzhugh-Nagumo model [30], that has been adapted to reproduce the characteristic temporal dynamics of the extracellular potassium during CSD, with a resting value of $4 \mathrm{mM}$, a peak value of $64 \mathrm{mM}$, and the plateau length matching the duration of the neuron activity disruption that follows the passage of the CSD (around 10 minutes, 31]).

Finally, a diffusion term accounts for the spatial propagation of the extracellular potassium wave across the cortex, and the complete model is a coupled 
reaction-diffusion $\mathrm{PDE} / \mathrm{ODE}$ system that reads:

$$
\begin{aligned}
\frac{\partial u}{\partial t} & =\operatorname{div}(D \nabla u)-F(u, w) \\
\frac{\partial w}{\partial t} & =G(u, w) \\
F(u, w) & =\eta_{1}\left(u-u_{0}\right)\left(1-\frac{u}{u_{t h}}\right)\left(1-\frac{u}{u_{p}}\right)+\eta_{2}\left(u-u_{0}\right) w \\
G(u, w) & =\gamma\left(u-u_{0}-\eta_{3} w\right),
\end{aligned}
$$

where $u(x, t)$ is the extracellular potassium concentration in $x$ at time $t \geq 0$, $w(x, t)$ is a dimensionless recovery variable, $u_{t h}$ and $u_{p}$ are threshold and peak values for $u, u_{0}$ is the resting concentration, $D \in \mathbb{R}^{3 \times 3}$ is a diffusion tensor (depending on space, as it will be described in section 4), while $\eta_{1}, \eta_{2}, \eta_{3}$ and $\gamma$ are parameters, whose values are given in Table 1. The parameter values are tuned to match the characteristics of the spreading depression, namely the amplitude of the potassium wave and the duration of the disruption phase. The coupled PDE-ODE problem (1) is set on a computational domain $\Omega \times$ $(0, T), \Omega \subset \mathbb{R}^{3}$. A mathematically well posed problem is obtained by imposing initial conditions $u_{0}(x)=u(0, x)$ in $\Omega$ and boundary conditions on $\partial \Omega$. If the computational domain is a $2 \mathrm{D}$ surface $\Sigma \subset \mathbb{R}^{3}$ the classical divergence and gradient operators are replaced by their tangential counterparts $\operatorname{div}_{\Sigma}$ and $\nabla_{\Sigma}$. Boundary conditions are not necessary if the computational domain is a closed surface.

\begin{tabular}{c|l|l|l} 
Parameter & Description & Value & Units \\
\hline$u_{0}$ & resting value & 4 & $\mathrm{mM}$ \\
$u_{t h}$ & threshold parameter & 11.8 & $\mathrm{mM}$ \\
$u_{p}$ & peak value & 64 & $\mathrm{mM}$ \\
$\gamma$ & & $3.3333 \mathrm{e}-5$ & $\mathrm{mM}^{-1} \mathrm{~s}^{-1}$ \\
$\eta_{1}, \eta_{2}$ & & $0.2667,0.4806$ & $\mathrm{~s}^{-1}$ \\
$\eta_{3}$ & & 60 & $\mathrm{mM}$
\end{tabular}

Table 1: Parameters of the distributed model for extracellular potassium.

\subsection{Variational formulation}

Let us denote by $L^{2}(\Omega)$ and $H^{1}(\Omega)$ the classical spaces of square integrable functions on $\Omega$, and the classical Sobolev space on $\Omega$ over $\mathbb{R}$. The variational formulation of system (1) reads as follows.

Given $u_{0}, w_{0} \in L^{2}(\Omega)$, find $u \in W^{1,1}\left(0, T ; H^{1}(\Omega)\right)$, and $w \in L^{2}\left(0, T ; L^{2}(\Omega)\right)$ such that, $\forall t \in(0, T)$ 


$$
\begin{array}{ll}
\frac{\partial}{\partial t}(u(t), \phi)+a(u(t), \phi)+(F(u(t), w(t)), \phi)=0 & \forall \phi \in H^{1}(\Omega) \\
\frac{\partial}{\partial t}(w(t), \psi)=(G(u(t), w(t)), \psi) & \forall \psi \in L^{2}(\Omega),
\end{array}
$$

with suitable initial conditions on $u$ and $w$, as described in section 2 .

In equation $(2)$ we denote by (., .) the inner product in $L^{2}(\Omega)$

$$
(\eta, \chi)=\int_{\Omega} \eta \chi d x \quad \forall \eta, \chi \in L^{2}(\Omega),
$$

and by $a(.,$.$) the elliptic bilinear form on H^{1}(\Omega) \times H^{1}(\Omega)$

$$
a(\lambda, \mu)=\int_{\Omega}(\nabla \lambda)^{T} D(x) \nabla \mu d x \quad \forall \lambda, \mu \in H^{1}(\Omega),
$$

respectively. Several theoretical results on reaction-diffusion equations can be applied to equation (2). Owing to the fact that the bilinear form $a(\cdot, \cdot)$ is weakly coercive on $H^{1}(\Omega) \times H^{1}(\Omega)$, a detailed well-posedness analysis of this problem would follow the argument presented in [32] for an analogous system.

\section{Numerical approximation on individual geom- etry}

\subsection{Computational domain}

A crucial aspect in studying the behaviour of an individual brain relies on the use of personalized computational domains. The brain cortex is a thin layer of grey matter, characterized by a very large aspect ratio between its surface and its volume. At the spatial scale we are working, such layer can be approximated by a two-dimensional surface obtained from MRI T1 measurements. A priori the corpus callosum, an area of white matter connectivity fibres and not grey matter, could be excluded from the computational domain, but in this case boundary conditions should be imposed on the interface between corpus callosum and grey matter. The proper choice of these boundary conditions is a major problem: the natural choice of homogenous Neumann boundary conditions (i.e. no flux) is not viable, as these conditions model an isolated environment, while connections exist between the corpus callosum and the grey matter. Since a wrong choice of boundary conditions could impact the simulation results way more than the presence of the corpus callosum in the model, we made the conservative choice to include it in our computational domain. To summarize, the cortical geometry we used in this study is a closed surface $\Omega \subset \mathbb{R}^{3}$ that has been reconstructed from an MRI scan with FreeSurfer image analysis suite (http://surfer.nmr.mgh.harvard.edu/): for further details, see [33] and references therein. This data set was acquired and published in [34] and used in 
[21, and its acquisition was approved by the Ethics Committee at the Cruces University Hospital: all the methods employed were in accordance to approved guidelines. The data set corresponds to one healthy subject, male, age 28, and was acquired with a Philips Achieva 1.5T Nova scanner. The cortical STL geometry was obtained from a high-resolution anatomical MRI, acquired using a T1-weighted 3D sequence with the following parameters: $\mathrm{TR}=7.482 \mathrm{~ms}, \mathrm{TE}=$ $3.425 \mathrm{~ms}$; parallel imaging (SENSE) acceleration factor $=1.5$; acquisition matrix size $=256 \times 256 ; \mathrm{FOV}=26 \mathrm{~cm}$; slice thickness $=1.1 \mathrm{~mm} ; 170$ contiguous sections.

The computational mesh is a triangulation of the reconstructed cortex, consisting of 140,208 nodes and 280,412 triangles for the left hemisphere, and 139,953 nodes and 279,902 triangles for the right hemisphere.

\subsection{Semi-discrete formulation}

Let $\mathcal{T}_{h}$ be the triangulation of $\Omega \subset \mathbb{R}^{3}$ described in the previous section, namely $\Omega=\bigcup_{j=1}^{N} K_{j}$ (with $N=280,412$ for the left hemisphere, and $N=279,902$ for the right hemisphere), where each $K_{j} \in \mathcal{T}_{h}$ is the image of the reference triangle $E$, with vertices $(0,0,0),(1,0,0)$, and $(0,1,0)$, through an invertible affine map $T_{K_{j}}\left(K_{j}=T_{K_{j}}(E)\right)$. We define $h$ as the maximum diameter of the elements of the triangulation. The associated finite element spaces $X_{h}^{k}$ (see e.g. 35] for an introduction to finite element methods) is defined as

$$
X_{h}^{k}=\left\{\varphi_{h} \in C^{0}(\Omega) \mid \varphi_{h \mid K_{j}} \circ T_{K_{j}} \in \mathbb{P}_{k}(E)\right\}
$$

where $\mathbb{P}_{k}(E)$ is the space of polynomials of degree at most $k$ on $E$. In the numerical simulation presented in this paper we use piecewise linear $(k=1)$ elements.

A semi-discrete problem in space is then obtained by applying a Galerkin procedure, using as finite dimensional space $V_{h}=X_{h}^{k}$, and choosing a basis for $V_{h}$. Let then $\left\{\varphi_{i}\right\}_{i=1, \ldots, N_{h}}$ be the finite element basis, where $N_{h}=140,208$ for the left hemisphere, and $N_{h}=139,953$ for the right hemisphere. The semidiscrete unknowns are thus given by

$$
u_{h}(x, t)=\sum_{i=1}^{N_{h}} \boldsymbol{u}_{i}(t) \varphi(x) \quad w_{h}(x, t)=\sum_{i=1}^{N_{h}} \boldsymbol{w}_{i}(t) \varphi(x) .
$$

We define the semi-discrete unknown vectors $\boldsymbol{u}_{h}=\left[\boldsymbol{u}_{1}(t), \ldots, \boldsymbol{u}_{N_{h}}(t)\right]^{T}$ and $\boldsymbol{w}_{h}=\left[\boldsymbol{w}_{1}(t), \ldots, \boldsymbol{w}_{N_{h}}(t)\right]^{T}$, and we introduce the symmetric mass, $M=\left(m_{k l}\right)$, and stiffnes, $S=\left(s_{k l}\right)$, matrices, defined as

$$
m_{k l}=\sum_{j=1}^{N} \int_{K_{j}} \varphi_{k} \varphi_{l} d x \quad s_{k l}=\sum_{j=1}^{N} \int_{K_{j}}\left(\nabla \varphi_{k}\right)^{T} D(x) \nabla \varphi_{l} d x
$$

Numerical evaluation of the above integrals is obtained by means of a suitable quadrature rule. 
With these positions, the finite elements approximation of equation (1) is the solution of

$$
M \frac{\partial \boldsymbol{u}_{h}}{\partial t}+A \boldsymbol{u}_{h}+M F\left(\boldsymbol{u}_{h}, \boldsymbol{w}_{h}\right)=\mathbf{0},
$$

coupled with the semi-discrete formulation of the dynamics of the recovery variable

$$
\frac{\partial \boldsymbol{w}_{h}}{\partial t}=G\left(\boldsymbol{v}_{h}, \boldsymbol{w}_{h}\right)
$$

\subsection{Fully discrete approximation}

In order to have a fully discrete approximation of the problem, system (4)(5) must be integrated in time. For the sake of simplicity in presentation, we consider a fixed time step $\Delta t$, although time adaptive scheme can be considered as well. Let thus $t^{n}=n \Delta t$, for $n=0, \ldots, N_{t}$, be a discretisation of the time interval $(0, T)$ : we denote with superscript $n$ the variables computed at time $t^{n}$. With these positions, we integrate in time system (4)-(5) by means of a mixed implicit/explicit (IMEX) scheme: the linear diffusion term is discretized implicitly, while the nonlinear reaction term $F\left(\boldsymbol{u}_{h}, \boldsymbol{w}_{h}\right)$ is treated explicitly. The mass matrix $M$ can be lumped to diagonal form by standard techniques. Thanks to its specific form, the ordinary differential equation for the recovery variable can be linearised around the extracellular potassium concentration at the previous time step, and integrated exactly on $(0, \Delta t)$.

This allows us to decouple the PDE-ODE system by solving with respect to the recovery variable $\boldsymbol{w}_{h}^{n+1}$ first, given the extracellular potassium concentration at the previous time step $\boldsymbol{u}_{h}^{n}$, and then solving for $\boldsymbol{u}_{h}^{n+1}$

$$
M \frac{\boldsymbol{u}_{h}^{n+1}-\boldsymbol{u}_{h}^{n}}{\Delta t}+S \boldsymbol{u}_{h}^{n+1}+M F\left(\boldsymbol{u}_{h}^{n}, \boldsymbol{w}_{h}^{n+1}\right)=\mathbf{0} .
$$

With this choice (notice that one could solve for the extracellular potassium concentration first and update successively the recovery variable), the IMEX method to approximate system (4)-(5) requires to solve, at each time step, the linear system

$$
(M+\Delta t S) \boldsymbol{u}_{h}^{n+1}=M \boldsymbol{u}_{h}^{n}-\Delta t M F\left(\boldsymbol{u}_{h}^{n}, \boldsymbol{w}_{h}^{n+1}\right) .
$$

By letting $A:=M+\Delta t S$, the overall scheme can be summarized as follows.

$$
\begin{aligned}
\text { Given } \boldsymbol{u}_{h}^{0} \text { and } \boldsymbol{w}_{h}^{0}, \text { for } n & =0, \ldots, N_{t} \text { : } \\
\text { update: } & \boldsymbol{w}_{h}^{n+1}=\frac{\boldsymbol{u}_{h}^{n}-u_{0}}{\eta_{3}}+\left(\boldsymbol{w}_{h}^{n}-\frac{\boldsymbol{u}_{h}^{n}-u_{0}}{\eta_{3}}\right) \exp \left(-\eta_{2} \eta_{3} \Delta t\right) \\
\text { update: } & F_{h}^{n+1}=F\left(\boldsymbol{u}_{h}^{n}, \boldsymbol{w}_{h}^{n+1}\right) \\
\text { solve: } & A \boldsymbol{u}_{h}^{n+1}=M \boldsymbol{u}_{h}^{n}-\Delta t M F_{h}^{n+1} .
\end{aligned}
$$




\section{Model personalization via Diffusion Tensor Imag- ing data}

The use of an individual geometry as a computational domain, although necessary, is not sufficient to have an accurate patient-specific modeling of CSD propagation. Another highly individual feature resides in the anisotropic conductivity of the grey matter, and the proper identification of the associated tensor is of paramount importance. In this direction, we resort to locally derive the conductivity coefficients from Diffusion Tensor Imaging (DTI) data. DTI is an imaging technique based on Magnetic Resonance Imaging (MRI) that measures the diffusion of water molecules in different directions for every voxel of an MRI image. The underlying idea of DTI is that random movement of water molecules is influenced and governed by its surrounding tissue structure and geometric organisation. The water diffusion tensor obtained from DTI measurements is assumed to be a good approximation for the electrical conductivity tensor [22, 23, 24].

DTI data is normally given in the form of three positive eigenvalues $\lambda_{1}, \lambda_{2}, \lambda_{3}$ and three associated normalised and orthogonal eigenvectors $\boldsymbol{w}_{1}, \boldsymbol{w}_{2}, \boldsymbol{w}_{3}$ in each grid point or voxel. These eigenvalues and eigenvectors identify the principal directions and intensities of movement of water molecules. The eigenvectors are sorted according to the size of the eigenvalues, thus $\boldsymbol{w}_{1}$ describes the strongest direction of the movement. The diffusion in a three dimensional space can be expressed in the form of a diffusion tensor

$$
D=\lambda_{1} \boldsymbol{w}_{1} \boldsymbol{w}_{1}^{T}+\lambda_{2} \boldsymbol{w}_{2} \boldsymbol{w}_{2}^{T}+\lambda_{3} \boldsymbol{w}_{3} \boldsymbol{w}_{3}^{T}=\left(\begin{array}{ccc}
D_{x x} & D_{x y} & D_{x z} \\
D_{y x} & D_{y y} & D_{y z} \\
D_{z x} & D_{z y} & D_{z z}
\end{array}\right) .
$$

In general, such diffusion tensors in a given point are visually represented by an ellipsoid, where the eigenvectors identify the directions of the ellipsoid's semi-axes and the eigenvalues their respective length. In homogenous media this diffusion tensor is a multiple of the identity matrix and its representation is a sphere.

In Figure 1 we plot the DTI data in the nodes of the left hemisphere mesh described in the previous section. In the top row, a lateral and medial visualisations of the hemisphere provide a global view of the anisotropy: ellipsoids are coloured according to the size of the largest eigenvalue. Two levels of zoom of the lateral surface data are plotted in the bottom row. Figure 1 highlights how the anisotropy is not just local, but actually global (in terms of differences in shape, orientation and size between ellipsoids associated with different points).

\subsection{DTI data acquisition procedure}

The DTI data was processed with the FSL software (http://fsl.fmrib.ox. ac.uk/). First, an eddy current correction was applied to overcome the artefacts produced by head movement and to correct the changes produced by the variations in gradient field directions during the acquisition. The gradient directions 

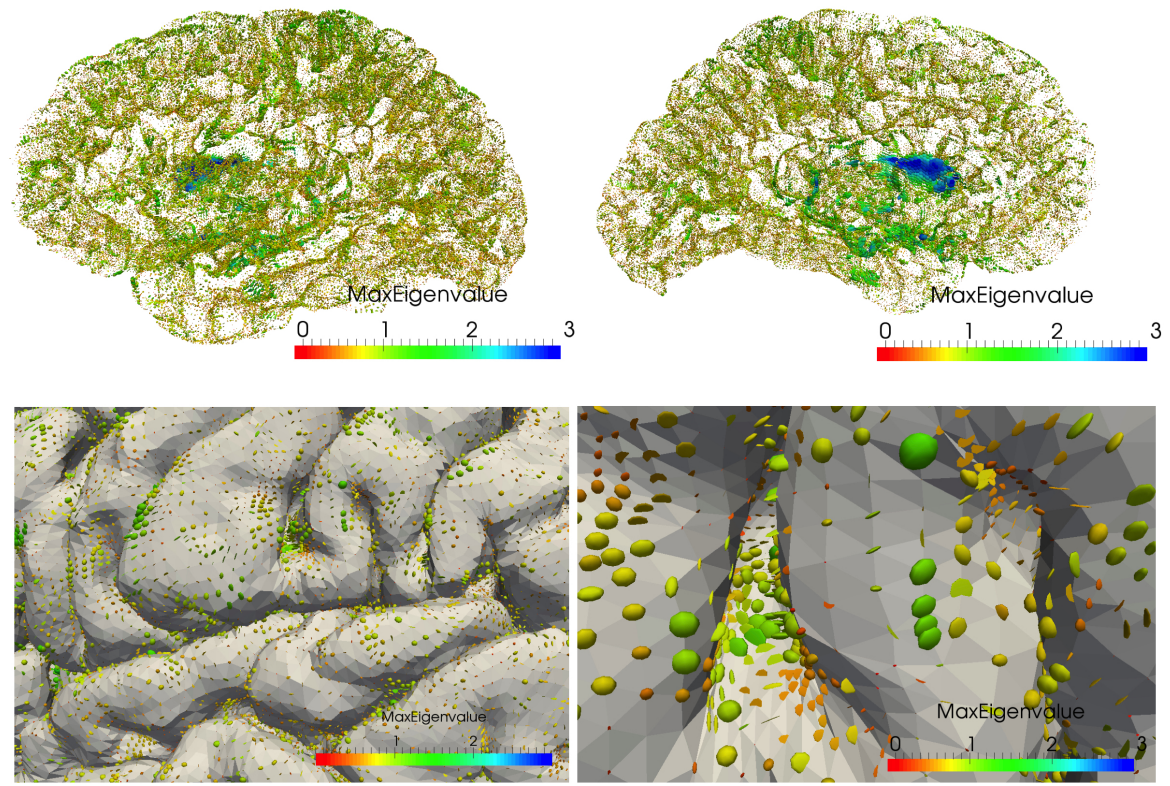

Figure 1: DTI data for the left hemisphere: ellipsoids colouring by to the size of the maximum eigenvalue. Top row: Lateral (left) and medial (right) surfaces of the DTI data. Bottom row: two levels of zoom of the lateral surface.

were corrected using the head movement matrices obtained from the previous step. A local fitting of the diffusion tensor was then applied to compute the tensor model at each voxel. For each voxel, the tensor model provided the 3 eigenvectors and the associated eigenvalues of the diffusion of water molecules within brain tissues.

To project the tensor data into the mesh, we first computed a non-linear transformation between the T1 image and the fractional anisotropy map. This transformation took as input a previous linear transformation using 12 degrees of freedom and took the mutual information as cost function. Then, we used the FreeSurfer (http://freesurfer.net/) function mri_vol2surf to project the diffusion data onto the brain mesh.

Here, similarly to what was done in previous works by some of the authors of this paper 34, 38, 39, we applied deterministic tractography, where only one possible principal direction can be assigned to a given voxel. This procedure does not account for crossing fibers. To accurately calculate crossing fibers from diffusion tensor imaging one needs to use a different acquisition protocol [40]. The acquisition parameters we used in here are not the most appropriate for $q$-space imaging, where higher $b$ values and higher number of gradient directions field have to be used [41. Potentially this could lead to a misrepresentation of the DTI data and thus biased or incorrect conductivity coefficients. However, 
the present work is a first study on the effect of diffusion, but we plan to use in the future higher resolution multi shell acquisitions to address the effect of crossing fibers on propagation patterns.

The complex workflow of data acquisition techniques to obtain DTI data is prone to creating artefacts, that can originate from, among others, the gradient system hardware, the pulse sequence and motion, but also from inter-operator variability. The main source of artefacts resides in the fact that DTI measurements have a low signal-to-noise ratio (SNR). Low SNR measurements are very sensitive to motion and, as a result, negative eigenvalues may appear in the dataset 42. The other main type of artefact is missing information, in consequence of the DTI data acquisition technique itself. Conventionally, DTI measurements are recorded to analyse the diffusion in the white matter. The data acquisition is thus often terminated as the border region of the white matter is reached. As a consequence, some values for the conductivity in the grey matter are missing and are per se complemented with zero, making both eigenvalues and eigenvectors vanish. Proper treatment of these artefacts is addressed in the next sections.

\subsection{DTI data and tissue anisotropy}

Three different kind of information on the tissue structure can be extracted from DTI data in each voxel [43]. The mean diffusivity describes the mean-squared displacement of molecules in a certain region or voxel, the main direction of diffusivity focuses on the orientation of the underlying structures, while the Degree of Anisotropy Index (DAI) measures the entity of the deviation from the isotropic case.

The mean diffusivity, giving a general evaluation of the diffusion, is defined as the mean of the diagonal of the diffusion tensor $D$ in (7):

$$
\mathrm{D}_{M}=\frac{\operatorname{Tr}(D)}{3}=\frac{D_{x x}+D_{y y}+D_{z z}}{3},
$$

whose average value and distribution across the cortex can provide valuable information about the overall diffusion behaviour and speed. In a given point in space, since the trace of a matrix is the sum of its eigenvalues, the value $\mathrm{D}_{M}$ actually represents the mean of the eigenvalues of the tensor $D$

$$
\mathrm{D}_{M}=\frac{1}{3}\left(\lambda_{1}+\lambda_{2}+\lambda_{3}\right)=\bar{\lambda} .
$$

Several ways to assess DAI have been proposed [43, 44]: we highlight here the volume ratio (VR), the relative anisotropy (RA), and the fractional anisotropy (FA).

The Volume Ratio (VR) describes the ratio between the ellipsoid volume and a sphere volume with the radius $\bar{\lambda}$ (or, equivalently, $\mathrm{D}_{M}$ ). It is defined as

$$
\mathrm{V}_{R}=\frac{\lambda_{1} \lambda_{2} \lambda_{3}}{\bar{\lambda}^{3}}=\frac{\operatorname{det} D}{\mathrm{D}_{M}^{3}},
$$


and ranges between 1 and 0 , where 1 is a fully isotropic situation.

The Relative Anisotropy (RA) represents the ratio of the anisotropic part of the diffusion tensor $D$ to its isotropic part. It is a normalized standard deviation defined as

$$
\mathcal{R}_{A}=\sqrt{\frac{\left(\lambda_{1}-\bar{\lambda}\right)^{2}+\left(\lambda_{2}-\bar{\lambda}\right)^{2}+\left(\lambda_{3}-\bar{\lambda}\right)^{2}}{3 \bar{\lambda}^{3}}},
$$

and ranges from 0 to $2^{1 / 2}$, where 0 represents a fully isotropic condition.

Finally, the Fractional Anisotropy (FA) expresses the normalized variance of the eigenvalues, and is defined as

$$
\mathcal{F}_{A}=\sqrt{\frac{3\left[\left(\lambda_{1}-\bar{\lambda}\right)^{2}+\left(\lambda_{2}-\bar{\lambda}\right)^{2}+\left(\lambda_{3}-\bar{\lambda}\right)^{2}\right]}{2\left(\lambda_{1}^{2}+\lambda_{2}^{2}+\lambda_{3}^{2}\right)}} .
$$

The fractional anisotropy $\mathcal{F}_{A}$ ranges between 0 and 1 , where again 0 represents a fully isotropic condition, and is the most commonly used DAI measurement.

In Figure 2 we plot the $\mathcal{F}_{A}$ values computed for both left (top row) and right (bottom row) hemispheres. We also plot normalised histograms displaying the relative frequency of the $\mathcal{F}_{A}$ values. We can infer that, in the original $3 \mathrm{D}$ data set, the majority of areas feature a mild anisotropy. Still, some areas show very high anisotropy levels, as it is the case within the corpus callosum.

\subsection{Local construction of the diffusion tensors}

The numerical simulation of CSD on the reconstructed brain cortex is a two dimensional problem, as the computational mesh is a triangulated surface in 3D. In order to recover a $2 \mathrm{D}$ tensor from the $3 \mathrm{D}$ DTI data, the diffusion tensor is built locally on each element of the triangulation by intersecting the $3 \mathrm{D}$ diffusion ellipsoids with the plane identified by the triangle $K$ at hand. The procedure consists in 2 step. First, the triangle under consideration and the ellipsoids associated to its vertices are moved, by an isometric transformation of $\mathbb{R}^{3}$, to the $x y$-plane $z=0$. The isometry ensures that all angles and distances are maintained. We then intersect the transformed ellipsoids with the plane $z=0$, to identify the resulting $2 \mathrm{D}$ tensors. In the rest of this section we describe in detail the procedure. We point out that a similar procedure with minimal modifications can be devised in the case the cortical surface is discretized by quadrilateral elements instead of triangular ones.

In Figure 3 (top left) we show a generic element $K$ of the triangulation and the ellipsoid from DTI data in vertex $P_{1}$. The local numbering of the vertices in the triangle follows the one provided by the adjacency matrix of the mesh. Following this local numbering, we consider a basis for $\mathbb{R}^{3}$ composed by the vectors $\boldsymbol{v}_{1}=\overline{P_{1} P_{2}}, \boldsymbol{v}_{2}=\overline{P_{1} P_{3}}$, and the unit vector $\boldsymbol{v}_{3}=(0,0,1)$, that we assume not to belong to the plane. In the case the vector $(0,0,1)$ belongs to the plane of the triangle, it is enough to choose another vector from the canonical basis in $\mathbb{R}^{3}$ not belonging to the plane and modify what follows accordingly. We 

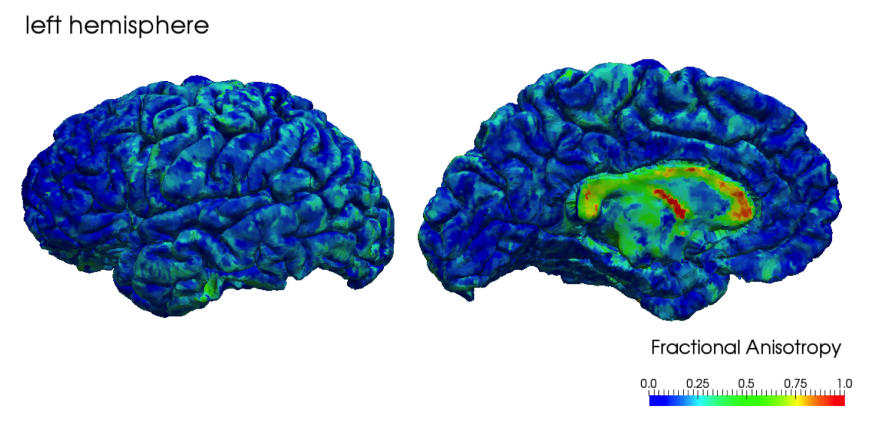

right hemisphere

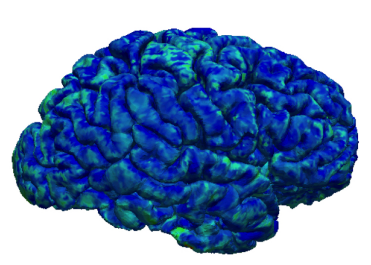

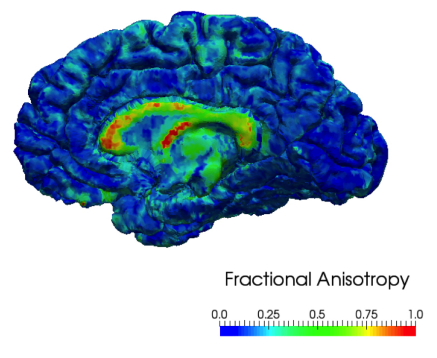
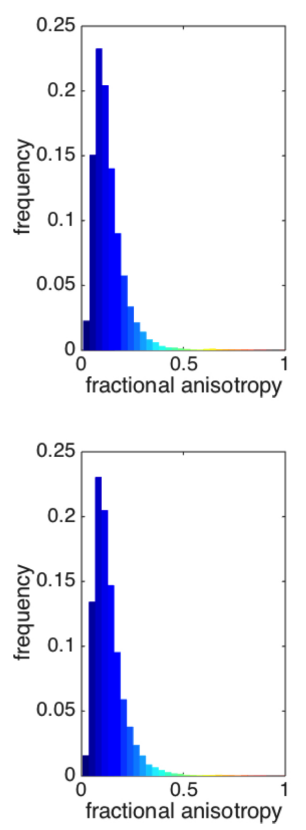

Figure 2: Fractional Anisotropy of the three dimensional DTI data and a normalised histogram displaying the probability density of the $\mathcal{F}_{A}$ values for the left (top) and right (bottom) hemisphere.

denote by $\langle\cdot, \cdot\rangle$ the scalar product in $\mathbb{R}^{3} \times \mathbb{R}^{3}$. The isometry is defined such that the original triangle is moved on the plane $z=0$, aligning the vector $\boldsymbol{v}_{1}$ with the $x$-axis, as shown in Figure 3 (top: left to right).

The ellipsoid representing the three dimensional DTI data in point $P_{j}(j=$ $1,2,3)$ is given by

$$
\left(X-P_{j}\right)^{T} W_{j} \Lambda_{j}^{-2} W_{j}^{T}\left(X-P_{j}\right)=1
$$

where $W_{j}=\left[\boldsymbol{w}_{1}, \boldsymbol{w}_{2}, \boldsymbol{w}_{3}\right]$ is the matrix whose columns are the eigenvectors in point $P_{j}$, while $\Lambda_{j}=\operatorname{diag}\left(\lambda_{1}, \lambda_{2}, \lambda_{3}\right)$ is the diagonal matrix of the eigenvalues in point $P_{j}$. We denote by $T \in \mathbb{R}^{3 \times 3}$ the matrix associated with the isometric transformation, and by $\widehat{P}_{j}=T P_{j}$ the transformed vertexes of the triangle: notice that $\widehat{P}_{1}=(0,0,0)$, while we have $\widehat{P}_{j}=\left(x_{j}, y_{j}, 0\right)(j=2,3)$. We provide an explicit description of $T$ at the end of this section. The equation of the image of the ellipsoid in $\widehat{P}_{j}$, under the transformation $T$ is then given by

$$
\left(X-\widehat{P}_{j}\right)^{T}\left(T W_{j}\right) \Lambda_{j}^{-2}\left(T W_{j}\right)^{T}\left(X-\widehat{P}_{j}\right)=1 .
$$

Let $B_{j}=\left(T W_{j}\right) \Lambda_{j}^{-2}\left(T W_{j}\right)^{T}$. By intersecting the ellipsoid 10$)$ with the plane 

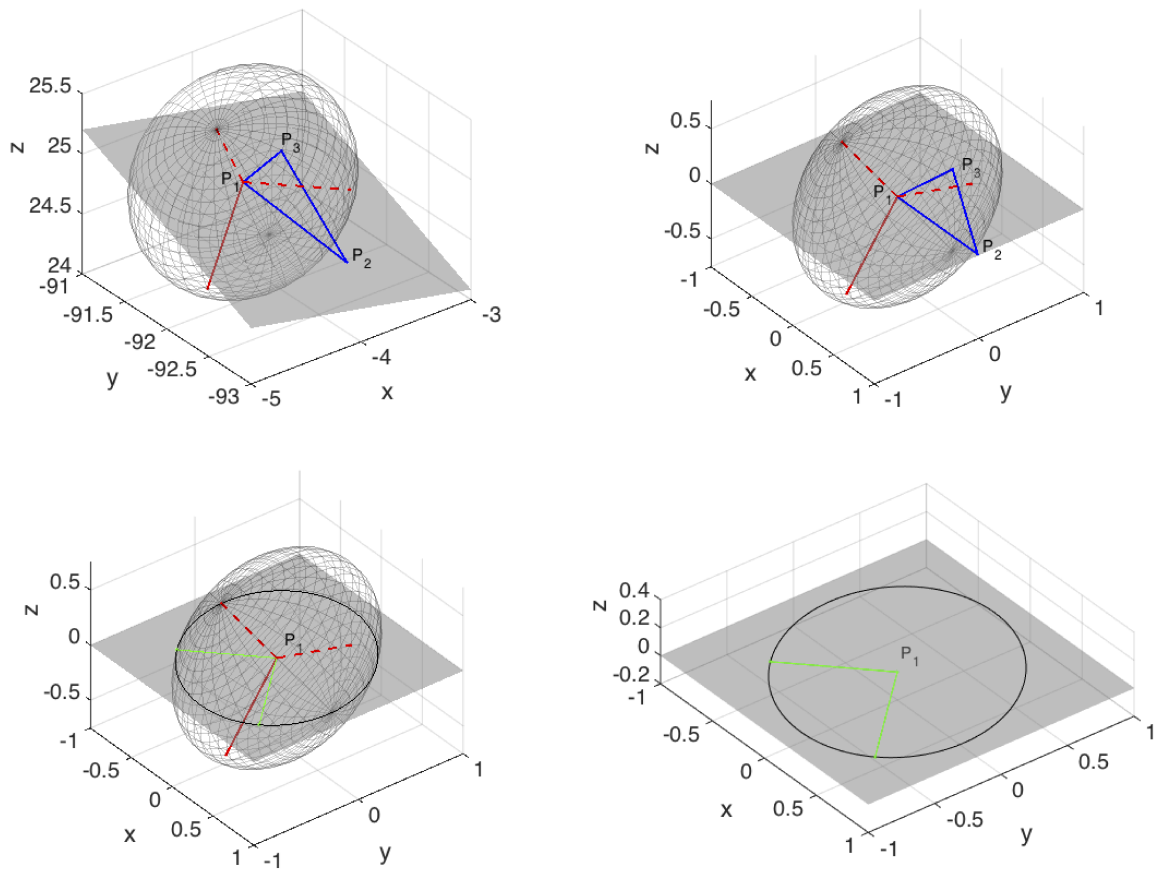

Figure 3: Top left: a generic element of the triangulation (in blue), and the ellipsoid from DTI data in vertex $P_{1}$. The plane identified by the triangle is shown in grey. Top right: the triangle and the ellipsoid associated with $P_{1}$ after the transformation. Bottom left: intersection between the transformed ellipsoid and the plane $\{z=0\}$ Bottom right: the main diffusion directions (in green) on the plane $\{z=0\}$ and the ellipse (in black) associated with the $2 \mathrm{D}$ conductivity tensor. For the sake of visualisation, in all the above figures, vector lengths have been magnified by a factor 1000 .

$z=0$, we obtain the ellipse

$$
\left[\begin{array}{l}
x-x_{j} \\
y-y_{j}
\end{array}\right]^{T} \widehat{B}_{j}\left[\begin{array}{l}
x-x_{j} \\
y-y_{j}
\end{array}\right]=1
$$

where we denoted by $\widehat{B}_{j} \in \mathbb{R}^{2 \times 2}$ the principal submatrix of $B_{j}$ of order 2 , obtained discarding the third row and the third column.

For each vertex of the triangle $\widehat{P}_{j}$, let $\left(\xi_{1, j}, \widehat{\boldsymbol{w}}_{1, j}\right)$ and $\left(\xi_{2, j}, \widehat{\boldsymbol{w}}_{2, j}\right)$ be the eigenvalues/eigenvectors pairs of $\widehat{B}_{j}$ (with normalized eigenvectors): the ellipse semi-axes are oriented along $\widehat{\boldsymbol{w}}_{1, j}$ and $\widehat{\boldsymbol{w}}_{2, j}$, and their lengths are given by $\mu_{1, j}=1 / \sqrt{\xi_{1, j}}$, and $\mu_{2, j}=1 / \sqrt{\xi_{2, j}}$.

We let

$$
\mu_{j}^{l}=\max \left(\mu_{1, j}, \mu_{2, j}\right), \quad \quad \mu_{j}^{t}=\min \left(\mu_{1, j}, \mu_{2, j}\right),
$$


and we denote with $\boldsymbol{p}_{j}$ and $\boldsymbol{q}_{j}$ the eigenvectors associated with $\mu_{j}^{l}$, and $\mu_{j}^{t}$, respectively. By construction, $\boldsymbol{p}_{j}$ corresponds to the major direction of the diffusion in 2D, whereas $\boldsymbol{q}_{j}$ is the minor direction of the diffusion (Figure 3 , bottom right). Notice that for an isotropic point $P_{j}$, as the intersection of a sphere with any plane through its centre is a circle, one has $\mu_{j}^{l}=\mu_{j}^{t}$. Since the diffusion tensors are built at the triangle level, additional information from DTI data can be obtained by considering the mean diffusivity and the fractional anisotropy associated with each triangle: the natural choice is to consider such values in its centroid. In the numerical simulations we chose $\mathbb{P}_{1}$ finite elements, and we can assume that both the orientation and the size of the diffusion coefficients change linearly across the triangle $K$. Thus, the diffusion coefficents in the centroid, that we denote by $\mu_{K}^{l}$ and $\mu_{K}^{t}$, can be interpolated as

$$
\mu_{K}^{l}=\frac{1}{3} \sum_{j=1}^{3} \mu_{j}^{l} \quad \text { and } \quad \mu_{K}^{t}=\frac{1}{3} \sum_{j=1}^{3} \mu_{j}^{t} .
$$

Owing to 12 , the $2 \mathrm{D}$ fractional anisotropy and the mean diffusivity of the triangle $K$ are given by

$$
\Phi_{K}=\frac{\mu_{K}^{l}-\mu_{K}^{t}}{\sqrt{\left(\mu_{K}^{l}\right)^{2}+\left(\mu_{K}^{t}\right)^{2}}} \quad \text { and } \quad M_{K}=\frac{\mu_{K}^{l}+\mu_{K}^{t}}{2},
$$

respectively. We denote by $M_{\text {mean }}$ the mean of $M_{K}$ over all the elements of the triangulation.

With these positions, we can finally define the $2 \mathrm{D}$ diffusion tensor, in the vertex $P_{j}(j=1,2,3)$ of the triangle $K$, as

$$
D_{j}=\frac{\delta}{M_{\text {mean }}}\left(\mu_{j}^{l} \boldsymbol{p}_{j} \boldsymbol{p}_{j}^{T}+\mu_{j}^{t} \boldsymbol{q}_{j} \boldsymbol{q}_{j}^{T}\right),
$$

where $\boldsymbol{p}_{j}$ and $\boldsymbol{q}_{j}$ are the principal diffusion directions in $P_{j}$.

The diffusion coefficient $\delta$ in (14) can be tuned to adapt the velocity of the extracellular potassium wave across the cortex to a propagation time that is comparable to the one of CSD. In the case real measurements were available, the parameter $\delta$ could actually be estimated through a data assimilation procedure (see, e.g., [36, 37]).

We conclude the section by describing in detail how to determine the matrix $T$ associated with the isometry, through its action on the basis $\left\{\boldsymbol{v}_{1}, \boldsymbol{v}_{2}, \boldsymbol{v}_{3}\right\}$. We denote by $T_{i j}$ the $(i, j)$-th entry of the transformation matrix $T$. Isometrically moving the triangle on the $z=0$ plane amounts to impose

$$
T \boldsymbol{v}_{1}=\left[\begin{array}{c}
\left\|\boldsymbol{v}_{1}\right\| \\
0 \\
0
\end{array}\right], \quad \quad T \boldsymbol{v}_{2}=\left[\begin{array}{c}
\left\|\boldsymbol{v}_{2}\right\| \cos \alpha \\
\left\|\boldsymbol{v}_{2}\right\| \sin \alpha \\
0
\end{array}\right],
$$

where

$$
\cos \alpha=\frac{\left\langle\boldsymbol{v}_{1}, \boldsymbol{v}_{2}\right\rangle}{\left\|\boldsymbol{v}_{1}\right\|\left\|\boldsymbol{v}_{2}\right\|} .
$$


As $\boldsymbol{v}_{3}=(0,0,1)$ we have

$$
T \boldsymbol{v}_{3}=\left[\begin{array}{l}
T_{13} \\
T_{23} \\
T_{33}
\end{array}\right],
$$

and we need 3 conditions to identify entries $T_{13}, T_{23}$, and $T_{33}$. The isometry assumption on $T$ entails $\left\|\widehat{\boldsymbol{v}}_{3}\right\|=1$, and we recover the two other conditions by imposing the conservation of the angles between the basis vectors $\left\{\boldsymbol{v}_{1}, \boldsymbol{v}_{2}, \boldsymbol{v}_{3}\right\}$.

Since

$$
\frac{\left\langle\boldsymbol{v}_{1}, \boldsymbol{v}_{3}\right\rangle}{\left\|\boldsymbol{v}_{1}\right\|\left\|\boldsymbol{v}_{3}\right\|}=\frac{\boldsymbol{v}_{1}(3)}{\left\|\boldsymbol{v}_{1}\right\|} \quad \frac{\left\langle\boldsymbol{v}_{2}, \boldsymbol{v}_{3}\right\rangle}{\left\|\boldsymbol{v}_{2}\right\|\left\|\boldsymbol{v}_{3}\right\|}=\frac{\boldsymbol{v}_{2}(3)}{\left\|\boldsymbol{v}_{2}\right\|}
$$

where $\boldsymbol{v}_{j}(i)$ denotes the $i$-th component of vector $\boldsymbol{v}_{j}$, the entries of the third column of $T$ are easily obtained as:

$T_{13}=\frac{\boldsymbol{v}_{1}(3)}{\left\|\boldsymbol{v}_{1}\right\|}, \quad T_{23}=\left(\frac{\boldsymbol{v}_{2}(3)}{\left\|\boldsymbol{v}_{2}\right\|}-\frac{\boldsymbol{v}_{1}(3)}{\left\|\boldsymbol{v}_{1}\right\|} \cos \alpha\right) \frac{1}{\sin \alpha}, \quad T_{33}=\sqrt{1-T_{13}^{2}-T_{23}^{2}}$.

By backward substitution in (15), a simple algebra shows that the remaining entries of $T$ can be obtained by solving the linear systems

$$
\begin{aligned}
& {\left[\begin{array}{ll}
\boldsymbol{v}_{1}(1) & \boldsymbol{v}_{1}(2) \\
\boldsymbol{v}_{2}(1) & \boldsymbol{v}_{2}(2)
\end{array}\right]\left[\begin{array}{l}
T_{11} \\
T_{12}
\end{array}\right]=\left[\begin{array}{c}
\left\|\boldsymbol{v}_{1}\right\|-\boldsymbol{v}_{1}(3) T_{13} \\
\left\|\boldsymbol{v}_{2}\right\| \cos \alpha-\boldsymbol{v}_{2}(3) T_{13}
\end{array}\right]} \\
& {\left[\begin{array}{ll}
\boldsymbol{v}_{1}(1) & \boldsymbol{v}_{1}(2) \\
\boldsymbol{v}_{2}(1) & \boldsymbol{v}_{2}(2)
\end{array}\right]\left[\begin{array}{l}
T_{21} \\
T_{22}
\end{array}\right]=\left[\begin{array}{c}
-\boldsymbol{v}_{1}(3) T_{23} \\
\left\|\boldsymbol{v}_{2}\right\| \sin \alpha-\boldsymbol{v}_{2}(3) T_{23}
\end{array}\right]} \\
& {\left[\begin{array}{ll}
\boldsymbol{v}_{1}(1) & \boldsymbol{v}_{1}(2) \\
\boldsymbol{v}_{2}(1) & \boldsymbol{v}_{2}(2)
\end{array}\right]\left[\begin{array}{l}
T_{31} \\
T_{32}
\end{array}\right]=\left[\begin{array}{l}
-\boldsymbol{v}_{1}(3) T_{33} \\
-\boldsymbol{v}_{2}(3) T_{33}
\end{array}\right] .}
\end{aligned}
$$

The crucial point in building the transformation $T$ is the unique solvability of the linear systems in (16), which is guaranteed as long as their matrix is not singular. Notice that the only possibility for it to be singular is that the first two component of $\boldsymbol{v}_{1}$ and $\boldsymbol{v}_{2}$ are aligned, but this option is ruled out by the assumption that $\boldsymbol{v}_{3}=(0,0,1)$ does not belong to the plane of the triangle. From the computational standpoint, however, although the matrix in 16 is not singular, it can be badly conditioned and trigger roundoff errors in the solution of the linear systems. To improve computational accuracy, one can check the conditioning of the matrix and, if necessary, center the transformation in one of the other two points of the triangle.

\subsubsection{Treatment of artefacts and missing data}

DTI data acquisition techniques are prone to creating artefacts, of various origin, that are principally constituted by missing values or negative eigenvalues. Missing values are normally a consequence of the DTI data acquisition technique itself, since DTI measurements are conventionally recorded to analyse the 
diffusion in white matter. The data acquisition is thus often terminated as the boundary of white matter is reached. As a consequence, some values for the diffusion in the grey matter are missing and are per se complemented with zero, making both eigenvalues and eigenvectors vanish. On the other hand, the major source of artefacts resides in the fact that DTI measurements have a low signalto-noise ratio (SNR). Low SNR measurements are very sensitive to motion and, as a result, negative eigenvalues may appear in the dataset [42]: we chose to treat such occurrences as missing values, and resort to the complementing strategy we describe in the following.

In Figure 4 we highlight (in red) the problematic mesh nodes in the database that we are considering in this paper: the spatial accumulation of artefacts in extremal portions of the cortex is evident, at the very top and front of the brain geometry. We can thus infer that, for the dataset at hand, the termination of data acquisition is probably the main reason behind missing data.
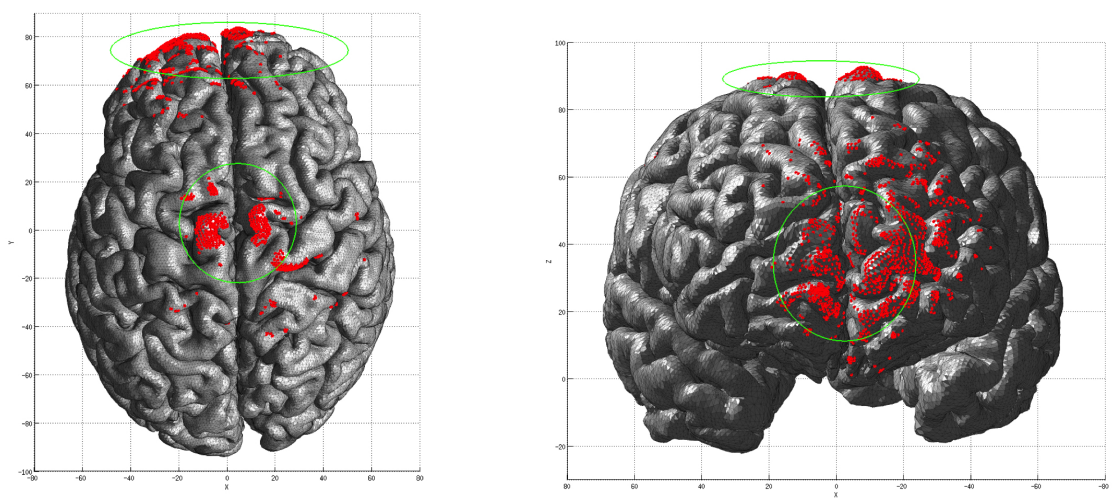

Figure 4: Spatial location of artefacts and missing DTI data points (in red) for both hemispheres.

In the grid points featuring missing values we complete the 3D dataset by assuming isotropic diffusion $\left(D=d \mathbb{I}_{3}\right)$. In order to identify a good candidate for the coefficient $d$, we consider a subdivision of the brain cortex into different regions of interest (ROIs): in particular, we rely on the anatomical subdivision of each hemisphere into 34 ROIs, which is a generalised version of the Brodmann atlas [45] (included in the MRIcro software http://www.mricro.com). In order to assign reasonable values in these critical points, we average the mean diffusivity $D_{M}$ of all points that lie in the same Broadmann region as the point, and do not feature missing values.

In the specific, by denoting with $\mathcal{R}$ the Broadmann region the critical point belongs to, with $\mathcal{P}_{\mathcal{R}}$ the set of points in $\mathcal{R}$ featuring data, by $\left|\mathcal{P}_{\mathcal{R}}\right|$ the cardinality 
of $\mathcal{P}_{\mathcal{R}}$, we assign the conductivity coefficient in the critical points as

$$
d=\sum_{P \in \mathcal{P}_{\mathcal{R}}} \frac{D_{M}}{\left|\mathcal{P}_{\mathcal{R}}\right|}
$$

\subsection{Computational aspects}

In the previous section we explained how to compute the diffusion tensors in the vertices of a triangle. However, when quadrature rules (see [46], chapter 8) are used to compute the stiffness matrix defined in (3), the diffusion tensors need to be evaluated also in other points of the triangle. We detail here how to compute the diffusion tensors in the midpoints of the sides and in the centroid of the triangle, as these are the quadrature points required by the third order Gaussian rule we use in our implementation. The basic idea is to compute the diffusion tensors in the sides' midpoints and centroid by interpolating both the orientation and the length of the semi-axes of the diffusion ellipses in the vertices. We point out that the following procedure can be easily extended to further additional points whenever a higher order quadrature rule is used.

Let $P_{i}$ and $P_{j}(i=1,2,3, j \neq i)$ be anisotropic points, and let $\vartheta$ be the angle between the major diffusion directions $\boldsymbol{p}_{i}$ and $\boldsymbol{p}_{j}$ in the two points, namely

$$
\vartheta=\arccos \left|\left\langle\boldsymbol{p}_{i}, \boldsymbol{p}_{j}\right\rangle\right|
$$

The principal diffusion directions in the midpoint between $P_{i}$ and $P_{j}$ are obtained by rotating vectors $\boldsymbol{p}_{i}$ and $\boldsymbol{q}_{i}$ by half the angle $\vartheta$ between $\boldsymbol{p}_{i}$ and $\boldsymbol{p}_{j}$. This rotation can be either clockwise or counterclockwise, but, for the sake of consistency, it has to be implemented in order to follow the shortest possible rotation path between $\boldsymbol{p}_{i}$ and $\boldsymbol{p}_{j}$. By introducing the rotation matrix $R$, defined as

$$
R=\left(\begin{array}{rr}
\cos (\vartheta / 2) & -\sin (\vartheta / 2) \\
\sin (\vartheta / 2) & \cos (\vartheta / 2)
\end{array}\right) \quad \text { or } \quad R=\left(\begin{array}{rr}
\cos (\vartheta / 2) & \sin (\vartheta / 2) \\
-\sin (\vartheta / 2) & \cos (\vartheta / 2)
\end{array}\right),
$$

according to the reciprocal position of the vectors $\boldsymbol{p}_{i}$ and $\boldsymbol{p}_{j}$ and the angle between them, the principal diffusion direction vectors in the midpoint are given by

$$
\boldsymbol{p}_{i j}=R \boldsymbol{p}_{i} \quad \text { and } \quad \boldsymbol{q}_{i j}=R \boldsymbol{q}_{i} .
$$

The eigenvalues $\mu_{i j}^{l}$ and $\mu_{i j}^{l}$ are computed by linearly interpolating between $\mu_{i}^{l}$ and $\mu_{j}^{l}$, and between $\mu_{i}^{t}$ and $\mu_{j}^{t}$ respectively:

$$
\mu_{i j}^{l}=\frac{\mu_{i}^{l}+\mu_{j}^{l}}{2} \quad \mu_{i j}^{t}=\frac{\mu_{i}^{t}+\mu_{j}^{t}}{2} .
$$

The diffusion tensors in the sides midpoints can then be easily computed by applying (14). The case when $P_{i}$ or $P_{j}$ is isotropic requires a little additional care. For illustration purpose, let $P_{j}$ be isotropic. In $P_{j}$ any direction can be 
considered the principal one, and we choose the principal directions coinciding with the ones of $P_{i}$. Thus, $\vartheta=0$, and we just interpolate the vector length.

For the diffusion tensor in the centroid, we already computed in 12 the diffusion coefficients in the centroid.

In order to have the associated eigenvectors, we consider the principal diffusion directions in the midpoint $P_{i j}$ of a side of the triangle $\left(\boldsymbol{p}_{i j}\right.$ and $\boldsymbol{q}_{i j}$, $i, j \in\{1,2,3\}, i \neq j)$, and the ones in the opposite vertex $P_{k}\left(\boldsymbol{p}_{k}\right.$ and $\boldsymbol{q}_{k}$, $k \notin\{i, j\})$. By letting $\phi$ be the angle between the major vectors $\boldsymbol{p}_{k}$ and $\boldsymbol{p}_{i j}$ in the two points,

$$
\phi=\arccos \left|\left\langle\boldsymbol{p}_{k}, \boldsymbol{p}_{i j}\right\rangle\right|,
$$

we rotate the principal diffusion directions $\boldsymbol{p}_{k}$ and $\boldsymbol{q}_{k}$ in the vertex by $2 / 3$ of the angle $\phi$. Also in this case the rotation must be performed along the shortest path for consistency. By introducing the rotation matrix $Q$, defined as

$Q=\left(\begin{array}{rr}\cos (2 \phi / 3) & -\sin (2 \phi / 3) \\ \sin (2 \phi / 3) & \cos (2 \phi / 3)\end{array}\right) \quad$ or $\quad Q=\left(\begin{array}{rr}\cos (2 \phi / 3) & \sin (2 \phi / 3) \\ -\sin (2 \phi / 3) & \cos (2 \phi / 3)\end{array}\right)$

depending on the reciprocal position of the vectors $\boldsymbol{p}_{k}$ and $\boldsymbol{p}_{i j}$, the principal diffusion direction vectors in the centroid are given by

$$
\boldsymbol{p}_{123}=Q \boldsymbol{p}_{k} \quad \text { and } \quad \boldsymbol{q}_{123}=Q \boldsymbol{q}_{k} .
$$

In the case when either $P_{i j}$ or $P_{k}$ is an isotropic point, the same procedure described before is applied. Again, the diffusion tensor in the centroid can then be easily computed by applying (14).

In Figure 5. we show the interpolation of the diffusion tensors on a triangle, both in the case of three anisotropic points (left) and the case of one isotropic point and two anisotropic ones (right). We plot the ellipses representing the 2D diffusion tensors, in red for the vertices, in green for the midpoints, and in blue for the centroid.

\section{$5 \quad$ Numerical results}

\section{$5.12 \mathrm{D}$ diffusion tensors}

The DTI data preprocessing from 3D to 2D tensors, that we introduced in the previous sections, is modifying the anisotropy characteristics of diffusion. In the $3 \mathrm{D}$ data set the majority of areas features a mild anisotropy for the underlying tissue; however, some areas, like the corpus callosum, show higher anisotropy levels. The 2D data set maintain the overall structure of a distributed mild anisotropy, although it exhibits a slightly larger amount of isotropic points (see Figure 6). This features depends strongly on the orientation of the 3D eigenvectors of the diffusion with respect to the surface. However, the areas featuring higher Fractional Anisotropy in the 3D dataset, in general, maintain this characteristic also after the processing of the data. 

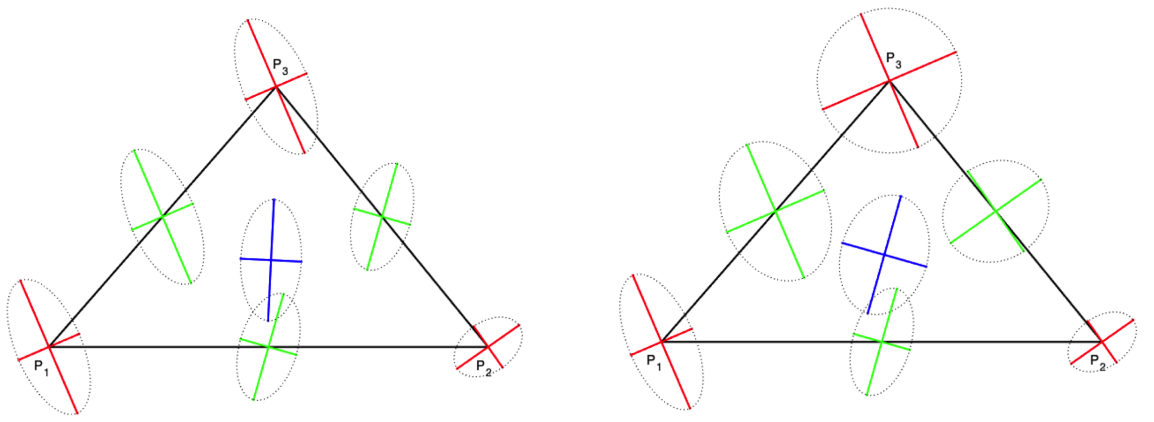

Figure 5: Interpolation of the diffusion ellipses on a triangle in the case of 3 anisotropic points (left) and the case of a vertex with isotropic diffusion (right): the axes of the diffusion ellipsoid are shown in red at the vertices, in green at the midpoints, and in blue at the centroid.

In Figure 7 we plot the mean diffusivity of the 2D transformed DTI data, for both the left (top) and right (bottom) hemisphere. In order to highlight their variability, the plotted data are normalized with respect to the mean across the whole cortex. From Figure 7 it can be appreciated that larger mean diffusivity values normally colocalize with sulci and with the corpus callosum. In the latter, high anisotropy values of white-matter tracts yield a much larger diffusion with respect to other cortical areas and the mesh geometry is much flatter: as a consequence, the simulated CSD propagation is expected to travel faster in the region.

Finally, we plot in Figure 8 the joint distribution of 2D Fractional Anisotropy and normalized mean diffusivity in the centroid of all triangles of the mesh, for both left and right hemisphere. Colors in Figure 8 represent density (bluelow, red-high) and one can observe the significant difference with respect to an isotropic situation, whose mean diffusivity and Fractional Anisotropy are identically 1 and 0 , respectively. For a clearer visual appreciation, the isotropic case is highlighted with a red bullet. Evidence from Figure 8 is a major advocate for the use of DTI-based diffusion coefficients.

\subsection{Simulation of CSD progression}

In this section we show the effectiveness of our method in simulating the CSD propagation across the whole cortex.

\subsubsection{Solver and preconditioning}

The numerical simulations of equations (1) are performed with a self-developed code in Matlab (MathWorks Inc., Natick, MA) with a uniform time step of $\Delta t=0.6$ seconds, and the parameter values given in Table 1 . 

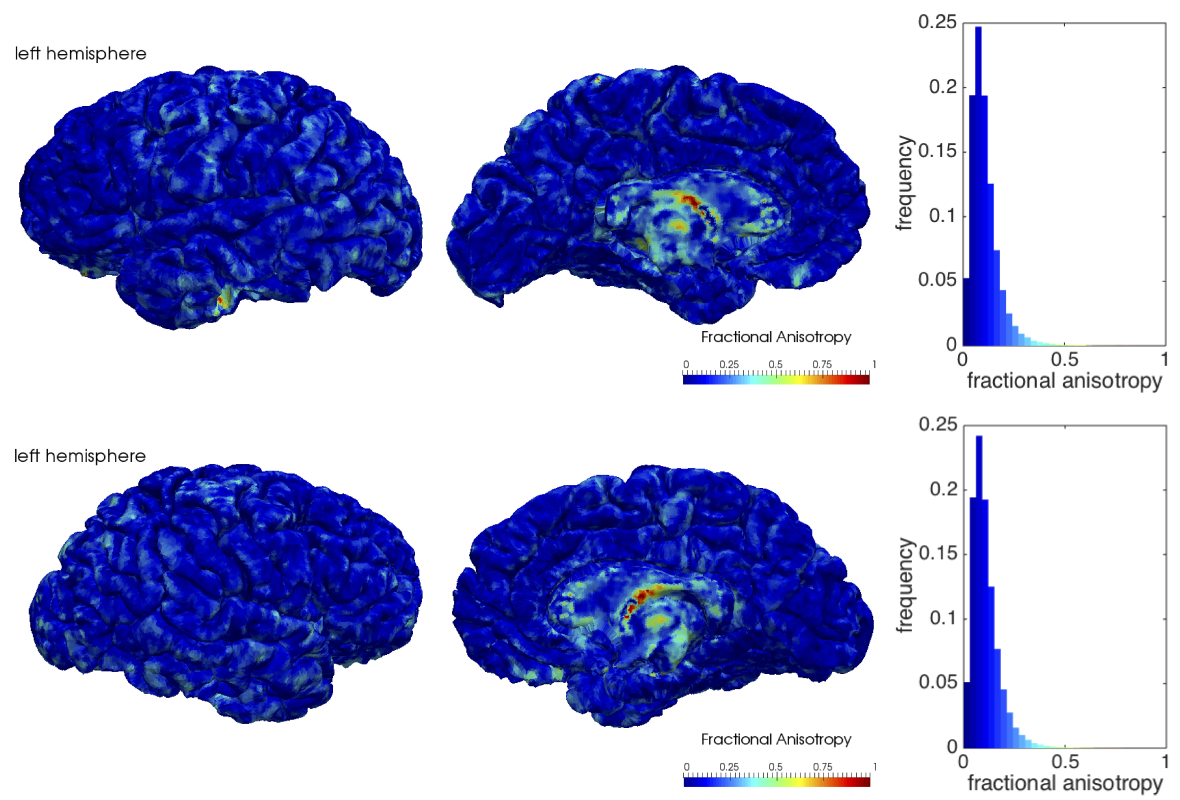

Figure 6: Fractional Anisotropy of the two dimensional DTI data and a normalised histogram displaying the probability density of the $\Phi_{K}$ values for the left (top) and right (bottom) hemisphere. 

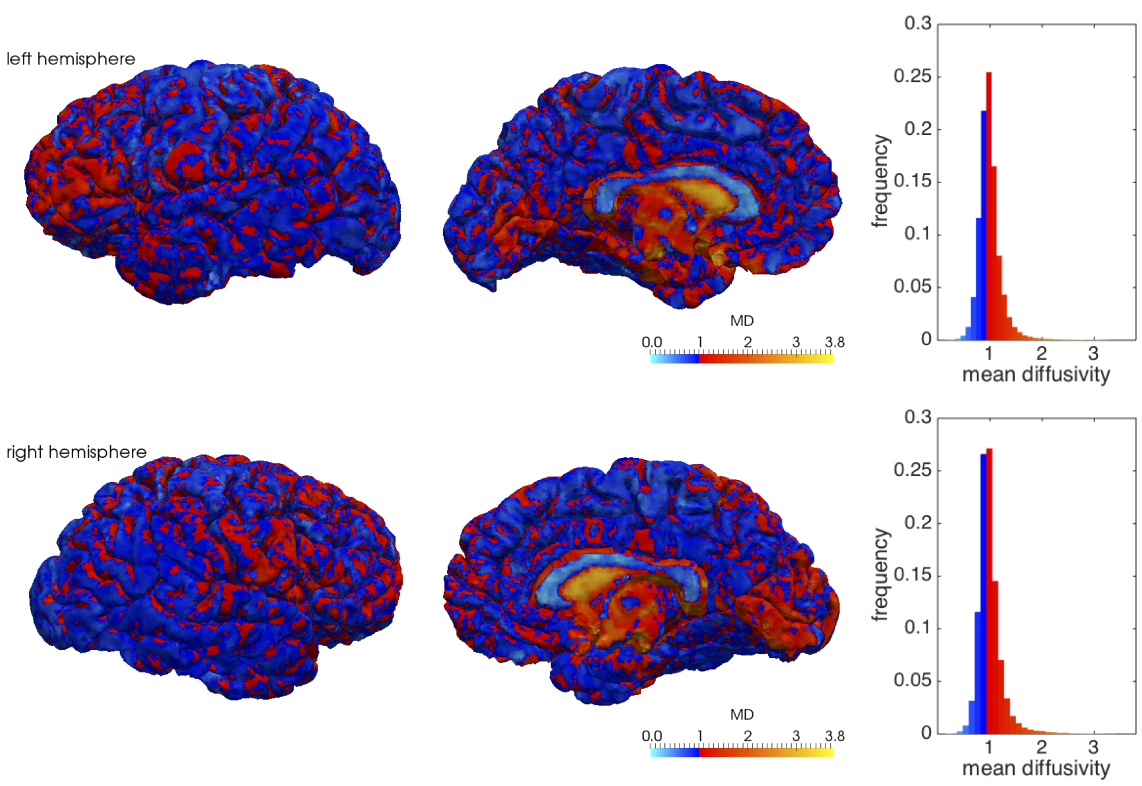

Figure 7: The mean diffusivity of the two dimensional DTI data of the cortex of the left (top) and right (bottom) hemisphere. Plotted data are normalised with respect to the average mean diffusivity across the whole cortical hemispheres.
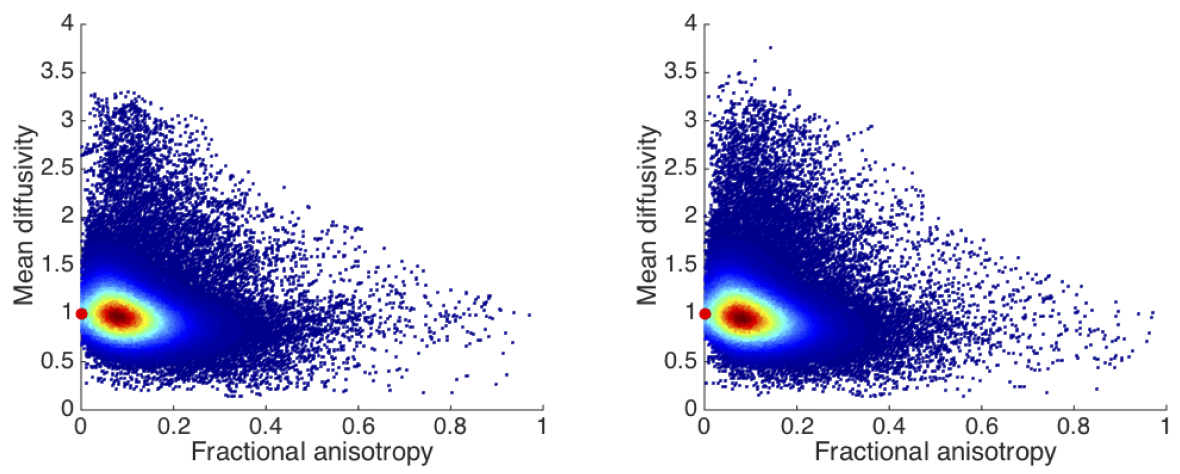

Figure 8: Joint distribution of the Fractional Anisotropy and the normalized mean diffusivity of the two dimensional DTI data for each triangle of the mesh (left and right hemisphere, respectively).

Colors represent density, while the red bullet highlights the isotropic case.

The IMEX scheme described in Section 3.3 requires to solve a linear system associated with a linear parabolic system, for which a vast literature on preconditioning is available [4]. The associated finite element matrix being symmetric, 
we solve system (6) with a Conjugate Gradient (CG) method, preconditioned by Incomplete Choleski factorization (IC), with pivoting and drop tolerance set at $10^{-6}$. Since the coefficients do not change in time during the simulation, so does the finite element matrix in (6): it is thus rentable to implement a low drop tolerance in the IC factorization, as the same preconditioner will be used along the large amount of time steps needed to simulate a whole CSD propagation. We consider as initial condition a fully depolarised lateraloccipital region, which corresponds to the area of the visual cortex where CSD is known to be originating from, and we run the simulation with the diffusion coefficients described in Section 3 until the whole cortex has been activated. The scaling coefficient $\delta=0.18 \mathrm{~mm}^{2} \mathrm{~s}^{-1}$ has been tuned to obtain a propagation time of around 20 minutes across the whole cortex, in agreement with the physics of the CSD. The stopping criterion for CG is set at $10^{-6}$, and a maximum number of iteration is set at 60 .
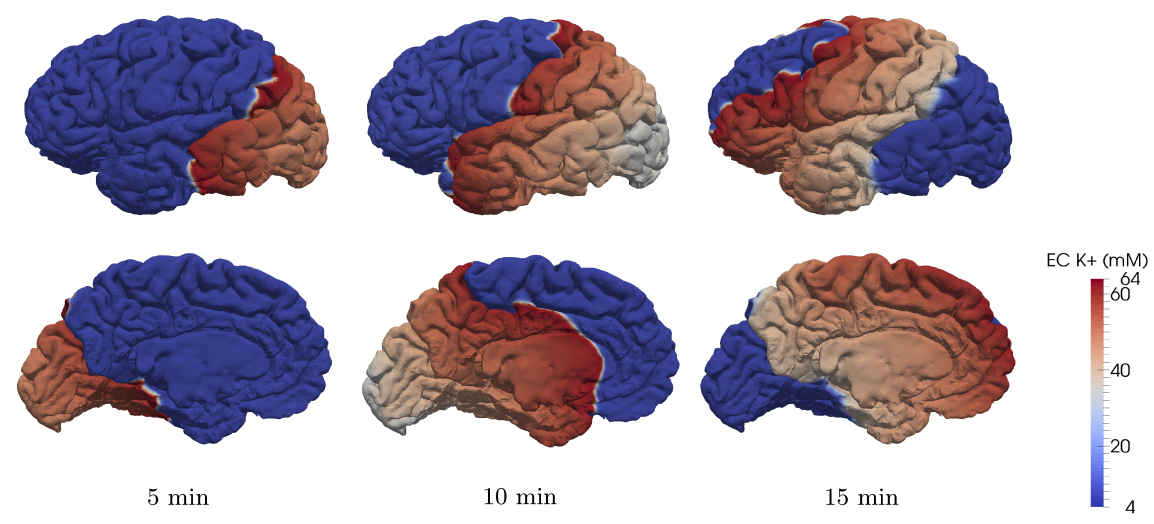

Figure 9: Lateral and medial views of extracellular potassium propagation dynamics on the left hemisphere at time $t=5 \mathrm{~min}, t=10 \mathrm{~min}$ and $t=15 \mathrm{~min}$.

In Figure 9 we plot the activation wavefronts on the left hemisphere at times $t=5 \mathrm{~min}, t=10 \mathrm{~min}$ and $t=16 \mathrm{~min}$. We plot in Figure 10 the iteration counts needed for convergence at each time step and relative residual at convergence during a full CSD simulation on the left hemisphere, for different drop tolerances in the IC factorization. We also collect in Table 2 the average iteration counts and average relative residual for both hemispheres for different values of the drop tolerance in IC. The combined effect of the low drop tolerance in the IC factorization, and the propagative nature of the solution of system (6) entails that choosing as initial guess, at time $t^{n+1}$, the solution at previous time step $u^{n}$, the preconditioned CG converges in very few iterations. Moreover, the single iteration needed in most cases for the $10^{-6}$ drop tolerance advocates for the use of more accurate preconditioning for the CSD simulation. 


\begin{tabular}{c|c|c|c|c}
\multirow{2}{*}{ drop tol. } & \multicolumn{2}{|c|}{ left hemisphere } & \multicolumn{2}{c}{ right hemisphere } \\
\cline { 2 - 5 } & iterations & rel. residual & iterations & rel. residual \\
\hline $10^{-6}$ & 1 & $2.1160 \mathrm{e}-8$ & 1 & $2.1680 \mathrm{e}-8$ \\
$10^{-5}$ & 1.0005 & $2.0239 \mathrm{e}-7$ & 1.0005 & $2.0378 \mathrm{e}-7$ \\
$10^{-4}$ & 2 & $3.0384 \mathrm{e}-9$ & 1.9768 & $2.2775 \mathrm{e}-8$ \\
$10^{-3}$ & 2.0266 & $1.6174 \mathrm{e}-7$ & 2.4679 & $3.6689 \mathrm{e}-7$
\end{tabular}

Table 2: Average number of iterations and average relative residual for different drop tolerances in the preconditioner computation for the left and right hemisphere.
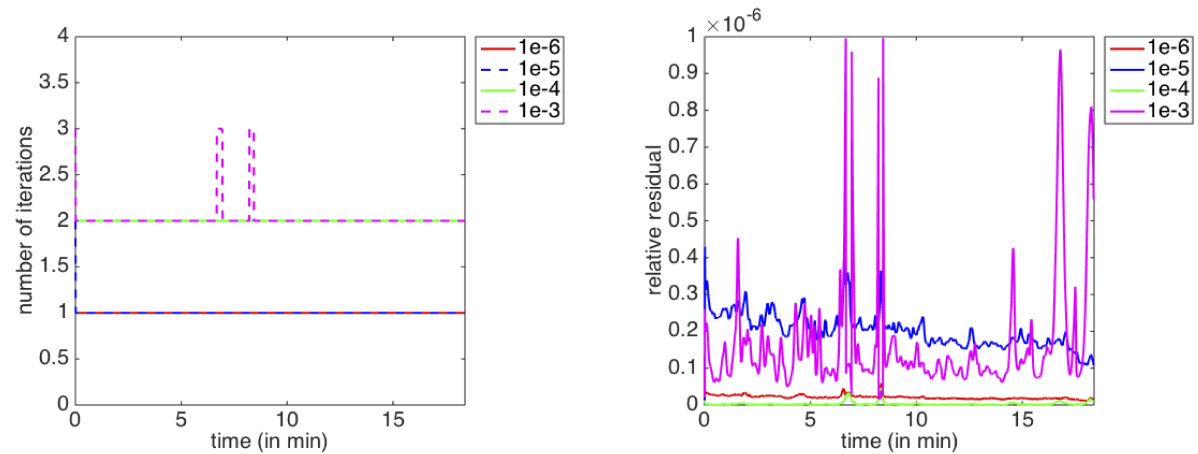

Figure 10: Iteration counts (left) and relative residual at convergence (right) along time, during a full CSD simulation on the left hemisphere for different drop tolerances in the computation of the preconditioner.

\subsubsection{Impact of tensor anisotropies on the CSD progression}

In this series of tests, in order to assess the effect of coefficients anisotropy on the progression of CSD across the cortex, we compare the DTI-based diffusion tensors with the isotropic diffusion used in [21]:

1. Isotropic diffusion (Iso). We assume, for all $K, \Phi_{K}=0$. The diffusion tensor in the generic point $P_{j}$ of the mesh is given by

$$
D_{j}=\delta \mathbb{I}_{2} .
$$

2. DTI-based anisotropic diffusion (DTI). We consider the conductivity tensor introduced in (14), that, in the generic point $P_{j}$, reads

$$
D_{j}=\frac{\delta}{M_{\text {mean }}}\left(\mu_{j}^{l} \boldsymbol{p}_{j} \boldsymbol{p}_{j}^{T}+\mu_{j}^{t} \boldsymbol{q}_{j} \boldsymbol{q}_{j}^{T}\right) .
$$

In order to compare the two different diffusion tensors introduced above, we consider the same scaling parameter $\delta=0.18 \mathrm{~mm}^{2} \mathrm{~s}^{-1}$. Again, we consider 
as initial condition a fully activated lateraloccipital region and we run the simulation until the whole cortex has been activated. From Figure 8, we expect differences in both propagation times and wavefront patterns. In Table 3 we collect to total activation time (namely the moment when the last grid point gets activated) for the two diffusion tensors, and for both hemispheres: the DTI-based anisotropic coefficient is resulting in a slower propagation across the whole cortex.

If, on the one hand, the overall propagation time across the cortex is comparable for the two different diffusion tensors, the activation patterns show, on the other hand, remarkable differences. We plot in Figure 11 the difference between the activation time for the isotropic diffusion and the DTI-based one for both left and right hemisphere. Difference in the activation patterns are not just visible between isotropic and DTI-based tensors, but also between the left and right hemisphere.

\begin{tabular}{c|c|c} 
Time $(\mathrm{min})$ & Iso & DTI \\
\hline Left hemisphere & 18.29 & 18.39 \\
Right hemisphere & 18.52 & 18.55
\end{tabular}

Table 3: Total activation time (minutes) for a extracellular potassium wave originating in the lateraloccipital region.

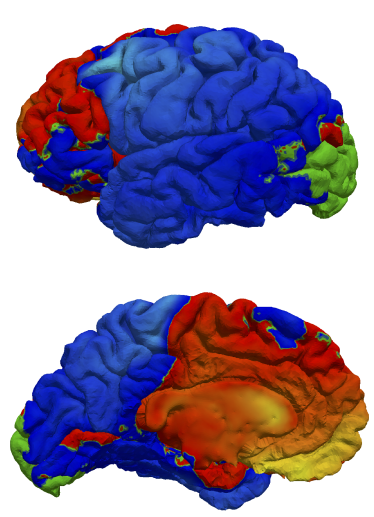

left hemisphere
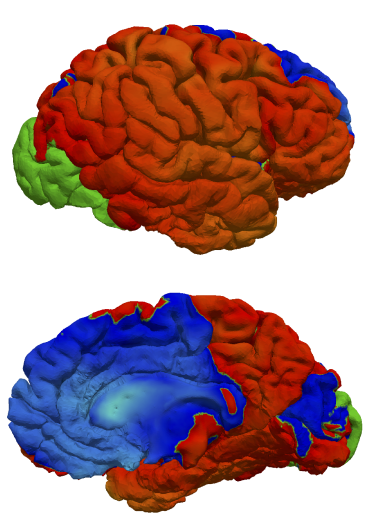

right hemisphere
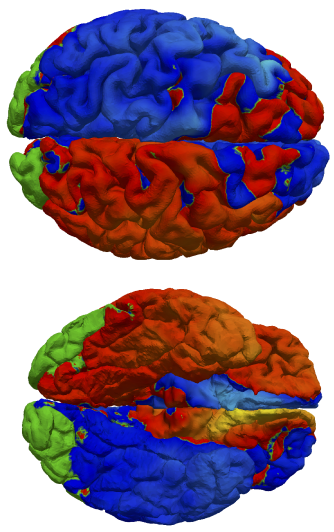

top/bottom view

time difference ( $\mathrm{min})$

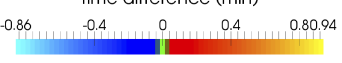

Figure 11: Difference between the activation times for the isotropic diffusion tensor and the DTI-based one. 


\subsection{Global assessment on the whole cortex}

In [21, some Quantities of Interest (QoI) have been introduced to globally assess dynamical symmetries on a cerebral cortex, both at intra and inter hemispherical level. Although CSD is originating in the visual cortex, in order to get a deeper understanding of the impact of the geometry on the propagation at a global scale, all 34 different regions in the Broadmann's subdivision of the cortex 45] are considered as starting area of an activation wave. In the specific, in each simulation one of these regions of the cortex is active as initial condition, and the activation times in all other points of the computational grid are recorded, identifying the minimum and maximum arrival times (the moment at which the first and the last point in a region are activated, respectively) in all the remaining 33 regions. The corpus callosum is the only region that is not considered as initially activated as it forms the intersection between the two hemispheres and obeys different rules for diffusion. The arrival times in the other regions are collected into four matrices, named $L_{m}, L_{M}, R_{m}$ and $R_{M}$, where $m$ and $M$ refer to minimum and maximum activation time, while $L$ and $R$ refer to the left and right hemisphere, respectively. In all these matrices, the $(i, j)$-th entry represents the arrival time in region $j$ of a potassium wave originating in region $i$. Following [21], the 34 regions are numbered by clustering them first according to the lobes, and, within lobes, according to the respective Euclidean distance of the regions' centroids. The matrices $L_{\tau}$ and $R_{\tau}(\tau=m, M)$ are the key ingredients in computing the QoI introduced in [21, and in this last section we highlight the impact of the choice of the conductivity tensor on such QoIs. We consider here, as an illustration, an example of an intra hemispherical asymmetry. By introducing the matrix $\Sigma$, defined as

$$
\Sigma_{i j}=\left\{\begin{array}{cc}
\frac{\left(L_{m}\right)_{i j}-\left(L_{m}\right)_{j i}}{\left(L_{m}\right)_{i j}} & \text { for } i>j \\
0 & \text { for } i=j \\
\frac{\left(R_{m}\right)_{j i}-\left(R_{m}\right)_{i j}}{\left(R_{m}\right)_{j i}} & \text { for } i<j,
\end{array}\right.
$$

the propagation symmetry properties of both hemispheres of a given cortex are collected into one single representation. Each $(i, j)$-th entry represents the difference in propagation time of a potassium wave traveling between regions $i$ and $j$ in the two opposite directions, normalised with respect to the propagation speed from $i$ to $j$. The lower triangular part of $\Sigma$ represents the left hemisphere, while its upper triangular part represents the right hemisphere.

In 21] it was shown that $\Sigma$ did not vanish already in the case of an isotropic diffusivity. Namely, the propagation speed of the activation wave on a cortical geometry does depend on the direction of travel, differently from what would happen on a sphere in the case of isotropic diffusivity. This result provides the first clear hint of the impact of the geometry in the propagation properties of the CSD. We plot in Figure 12 the matrices $\Sigma_{I s o}$ (left), and $\Sigma_{D T I}$ (right), computed from (18) with the isotropic and DTI-based diffusion coefficients introduced in the previous section. The normalization of the entries factors out 
the propagation speed and ensures us to deal with comparable quantities. We can observe from Figure 12 the significant variation triggered by the implementation of DTI-based coefficients with respect to an isotropic conductivity. The entries of $\Sigma_{I s o}$ and $\Sigma_{D T I}$ do not only differ in size, but in some cases also in sign, meaning that the fastest path between two regions can have different direction in the isotropic and the anisotropic case.
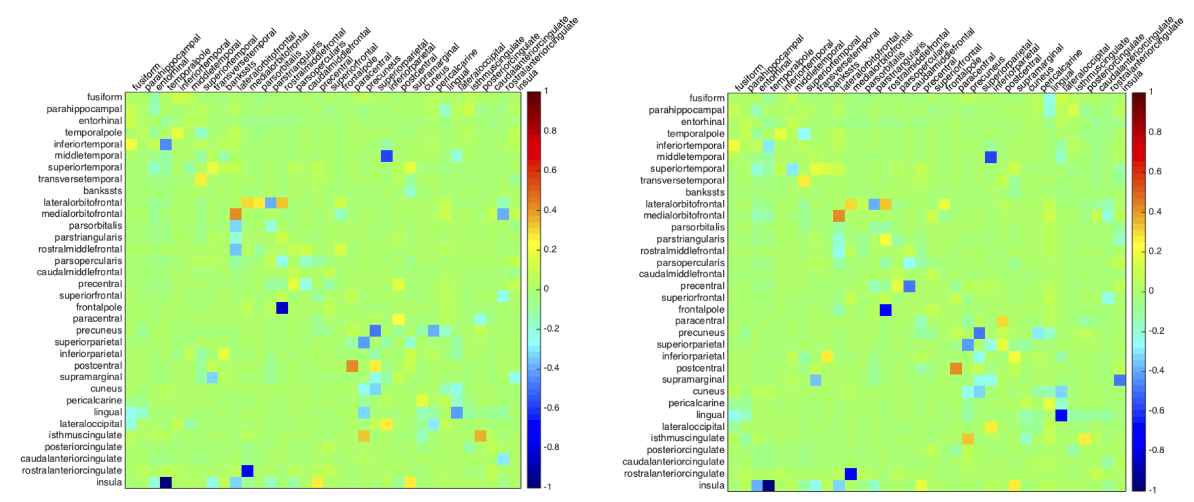

Figure 12: The matrices $\Sigma_{I s o}$ (left) and $\Sigma_{D T I}$ (right), computed with the isotropic and the DTI-based conductivity coefficients, respectively.

\section{Conclusions}

We introduced in this paper a patient-specific simulation technique for the propagation of Cortical Spreading Depression (CSD) on a real geometry. The computational domain is a cortical geometry reconstructed from MRI imaging. A higher level of personalization is added through the derivation of diffusion coefficients from 3D Diffusion Tensor Imaging (DTI) data. The algorithm to reduce the 3D DTI data to the 2D cortical surface is detailed in the paper: the diffusion tensors are computed triangle by triangle in a finite elements numerical approximation settings. Numerical results show differences in propagation time and, in particular, the wavefront shape with respect to an isotropic diffusion as considered in [21]. Moreover, relevant differences appear also in computing some of the QoIs introduced in 21] to globally assess symmetric and unsymmetric dynamical behaviour on the whole cortex. The main contribution of the proposed model resides in the use of a more detailed description of the tissue diffusivity: numerical simulations are expected to be a closer match to CSD propagation, especially in view of Figure 8 and Figure 11, at a minimal additional computational effort. The computational load of the proposed model is comparable with the one of the model introduced in [21. The only additional cost is associated with the pre-processing of the DTI data to compute the diffusion tensors, a procedure to be performed just once for any given dataset, not 
affecting the actual online computational time of the propagation: the number of linear systems to be solved is the same as in [21, with no additional computational issue. Finally, the proposed model can be a solid basis to build upon for tackling more complex problems. As an example, some experimental studies presented in the paper by Cruz and his collaborators [48] show the onset of a lactate wave following the passage of $\mathrm{K}^{+}$-induced CSD in mice: by possibly using a more accurate neuronal model (e.g. [49]) our method can be coupled with a metabolic model to reproduce such behaviour on real patient geometries.

\section{Acknowledgement}

This work was supported by the Bizkaia Talent and European Commission through COFUND under the grant BRAhMS - Brain Aura Mathematical Simulation (AYD-000-285), by the Basque Government through the BERC 20142017 program, and by the Spanish Ministry of Economics and Competitiveness MINECO through the BCAM Severo Ochoa excellence accreditation SEV-20130323 and the Spanish "Plan Estatal de Investigación, Desarrollo e Innovación Orientada a los Retos de la Sociedad" under Grant BELEMET - Brain ELEctroMETabolic modeling and numerical approximation (MTM2015-69992-R). JMC acknowledges financial support from Ikerbasque: The Basque Foundation for Science and Euskampus at UPV/EHU.

\section{References}

[1] Richter F, Lehmenkühler A. Cortical spreading depression (CSD): A neurophysiological correlate of migraine aura, Der Schmerz 2008; 22(5), doi: 10.1007/s00482-008-0653-9.

[2] de Tommaso M, Ambrosini A, Brighina F, Coppola G, Perrotta A, Pierelli F, Sandrini G, Valeriani M, Marinazzo D, Stramaglia S, Schoenen J. Altered processing of sensory stimuli in patients with migraine, Nat Rev Neurol 2014; 10: 144-155.

[3] Sanides F. Die Architektonik des menschlichen Stirnhirns, Monographien aus dem Gesamtegebiete der Neurologie und Psychiatrie (Springer Berlin) 1962; eds M. Müller, H. Spatz and P. Vogel, 98.

[4] Somjen GG. Mechanisms of spreading depression and hypoxic spreading depression-like depolarization. Physiological reviews 2001; 81(3):1065-96.

[5] Miura RM, Huang H, Wylie JJ. Mathematical approaches to modeling of cortical spreading depression, Chaos Dec 2013; 23(4), doi: $10.1063 / 1.4821955$.

[6] Pietrobon D, Moskowitz MA. Chaos and commotion in the wake of cortical spreading depression and spreading depolarizations. Nature Reviews Neuroscience 2014; 15(6):379-93. 
[7] Zandt BJ, ten Haken B, van Putten MJ, Dahlem MA. How does spreading depression spread? Physiology and modeling, Rev Neurosci. 2015; 26(2): 183-98, doi: 10.1515/revneuro-2014-0069.

[8] Tuckwell HC, Miura RM. A mathematical model for spreading cortical depression, Biophysical J. 1978; 23(2): 257-276, doi: 10.1016/S00063495(78)85447-2.

[9] Reggia JA, Montgomery D. A computational model of visual hallucinations in migraine, Computers in Biology and medicine 1996; 26(2): 133-141, doi: 10.1016/0010-4825(95)00051-8.

[10] Mori Y. A multidomain model for ionic electrodiffusion and osmosis with an application to cortical spreading depression. Physica D: Nonlinear Phenomena 2015 ; 308:94-108.

[11] Dahlem MA, Isele TM. A Transient Localized Wave Patterns and Their Application to Migraine, J. Math. Neurosci. 2013; 3(7), doi: 10.1186/21908567-3-7.

[12] Aguado-Sierra J, Krishnamurthy A, Villongco C, Chuang J, Howard E, Gonzales MJ, Omens J, Krummen DE, Narayan S, Kerckhoffs RC, McCulloch AD. Patient-specific modeling of dyssynchronous heart failure: a case study, Progress in biophysics and molecular biology 2011; 107(1): 147-155, doi: 10.1016/j.pbiomolbio.2011.06.014.

[13] Potse M, Krause D, Kroon W, Murzilli R, Muzzarelli S, Regoli F, Caiani E, Prinzen FW, Krause R, Auricchio A. Patient-specific modelling of cardiac electrophysiology in heart-failure patients, Europace 2014; 16(4): iv56iv61, doi: 10.1093/europace/euu257.

[14] Prakosa A, Malamas P, Zhang S, Pashakhanloo F, Arevalo H, Herzka DH, Lardo A, Halperin H, McVeigh E, Trayanova N, Vadakkumpadan F. Methodology for image-based reconstruction of ventricular geometry for patient-specific modeling of cardiac electrophysiology, Progress in biophysics and molecular biology 2014; 115(2-3): 226-234, doi: 10.1016/j.pbiomolbio.2014.08.009.

[15] Sermesant M, Chabiniok R, Chinchapatnam P, Mansi T, Billet F, Moireau P, Peyrat JM, Wong K, Relan J, Rhode K, Ginks M, Lambiase P, Delingette H, Sorine M, Rinaldi CA, Chapelle D, Razavi R, Ayache N. Patient-specific electromechanical models of the heart for the prediction of pacing acute effects in CRT: A preliminary clinical validation, Medical image analysis 2012, Elsevier, 16(1), pp. 201-215, doi: 10.1016/j.media.2011.07.003.

[16] Ukwatta E, Arevalo H, Rajchl M, White J, Pashakhanloo F, Prakosa A, Herzka DA, McVeigh E, Lardo AC, Trayanova NA, Vadakkumpadan F. Image-based reconstruction of three-dimensional myocardial infarct geometry for patient-specific modeling of cardiac electrophysiology, Medical physics 2015 ; 42(8): 4579-4590, doi: 10.1118/1.4926428.. 
[17] Vadakkumpadan F, Rantner LJ, Tice B, Boyle P, Prassl AJ, Vigmond E, Plank G, Trayanova N. Image-based models of cardiac structure with applications in arrhythmia and defibrillation studies, Journal of Electrocardiology 2009; 2(42): 157.e1-10, doi: 10.1016/j.jelectrocard.2008.12.003.

[18] Vázquez M, Arís R, Houzeaux G, Aubry R, Villar P, Garcia-Barnés J, Gil D, Carreras F. A massively parallel computational electrophysiology model of the heart, International Journal for Numerical Methods in Biomedical Engineering 2001; 27(12): 1911-1929, doi: 10.1002/cnm.1443.

[19] Dahlem MA, Schmidt B, Bojak I, Boie S, Kneer F, Hadjikhani N, Kurths J. Cortical hot spots and labyrinths: why cortical neuromodulation for episodic migraine with aura should be personalized, Front Comput Neurosci. $2015 ; \mathbf{9 ( 2 9 )}$, doi: 10.3389/fncom.2015.00029.

[20] Baysal U, Haueisen J, Durgut K, Demirci M. Simulating the propagation of spreading cortical depression (SCD) wavefront on human brain surface. Computers in biology and medicine 2007; 37(10):1446-54.

[21] Kroos JM, Diez I, Cortes JM, Stramaglia S, Gerardo-Giorda L. Geometry shapes propagation: assessing the presence and absence of cortical symmetries through a computational model of cortical spreading depression, Front. Comput. Neurosci. 2016; 10:6, doi: 10.3389/fncom.2016.00006.

[22] Tuch DS, Wedeen VJ, Dale AM, George JS, Belliveau JW. Conductivity Mapping of Biological Tissue Using Diffusion MRI, Annals New York Academy of Science 1999; 888: 314-316, doi: 10.1111/j.17496632.1999.tb07965.x.

[23] Tuch DS, Wedeen VJ, Dale AM, George JS, Belliveau JW. Conductivity tensor mapping of the human brain using diffusion tensor MRI, Proceedings of the National Academy of Sciences 2001; 98(20): 1697-11701, doi: 10.1073/pnas.171473898.

[24] Sekino M, Inoue Y, and Ueno S. Magnetic resonance imaging of mean values and anisotropy of electrical conductivity in the human brain. Neurol. Clin. Neurophysiol. 2004, vol. 55, pp. 1-5.

[25] Wang K, Zhu S, Mueller BA, Lim KO, Liu Z, He B. A new method to derive white matter conductivity from diffusion tensor MRI. IEEE Transactions on Biomedical Engineering. 2008; 55(10):2481-6.

[26] Cortes JM, Marinazzo D, Series P, Oram MW, Sejnowski TJ, van Rossum MCW. The effect of neural adaptation on population coding accuracy, Journal of Computational Neuroscience 2012; 32(3): 387-402, doi: 10.1007/s10827-011-0358-4.

[27] Izhikevich EM. Dynamical Systems in Neuroscience: The Geometry of Excitability and Bursting (Computational Neuroscience), MIT Press 2007. 
[28] Cressman JR Jr, Ullah G, Ziburkus J, Schiff SJ, Barreto E. The influence of sodium and potassium dynamics on excitability, seizure, and the stability of persistent states: I. Single neuron dynamics, J Comput Neurosci. 2009; 26(2): 159-170, doi: 10.1007/s10827-008-0132-4.

[29] Rogers JM, McCulloch AD. A collocation - Galerkin finite element model of cardiac action potential propagation, IEEE Trans Biomed Eng. 1994; 41(8): 743-757, doi: 10.1109/10.310090.

[30] FitzHugh R. Impulses and Physiological States in Theoretical Models of Nerve Membrane, Biophysical Journal 1961; 1(4): 445-466.

[31] Porooshani H, Porooshani AH, Gannon L, Kyle GM. Speed of progression of migrainous visual aura measured by sequential field assessment, NeuroOphthalmology 2004; 28(2): 101-105, doi: 10.1076/noph.28.2.101.23739.

[32] Colli Franzone P, Savarè G. Evolution equations semigroups and functional analysis: Degenerate evolution systems modeling the cardiac electric field at micro and macroscopic level, Progress in Nonlinear Differential Equations and Their Applications 2002; 50: 49-78.

[33] Fischl B. FreeSurfer, Neuroimage 2012, 62, pp. 774-781.

[34] Diez I, Bonifazi P, Escudero I, Mateos B, Muñoz MA, Stramaglia S. A novel brain partition highlights the modular skeleton shared by structure and function, Sci Rep 2015; 5: 10532, doi: 10.1038/srep10532.

[35] Quarteroni A, Valli A. Numerical Approximation of Partial Differential Equations, Springer-Verlag 1994, Berlin.

[36] D'Elia M, Mirabella L, Passerini T, Perego M, Piccinelli M, Vergara C, and Veneziani A. Applications of variational data assimilation in computational hemodynamics. In Modeling of Physiological Flows, pp. 363-394. Springer Milan, 2012.

[37] Delingette H, Billet F, Wong KC, Sermesant M, Rhode K, Ginks M, Rinaldi CA, Razavi R and Ayache N. Personalization of cardiac motion and contractility from images using variational data assimilation. IEEE transactions on biomedical engineering 2012; 59(1), pp.20-24.

[38] Amor TA, Russo R, Diez I, Mudnal P, Zirovich M, Stramaglia S, Cortes JM, de Arcangelis L and Chialvo DR. Extreme brain events: Higher order statistics of brain resting activity and its relation with structural connectivity. EPL , 2015, 111: 68007

[39] Alonso-Montes C, Diez I, Remaki L, Escudero I, Mateos B, Rosseel Y, Marinazzo D, Stramaglia S, Cortes JM. Lagged and instantaneous dynamical influences related to brain structural connectivity. Frontiers in Psychology, 2015, 6: 1024. 
[40] Tuch DS. Q-ball imaging. Magn Reson Med. 2004; 52(6):1358-72.

[41] Seo HS, Chang KH, Na DG, Kwon BJ, Lee DH. High b-value diffusion $(b=3000 \mathrm{~s} / \mathrm{mm} 2)$ MR imaging in cerebral gliomas at $3 \mathrm{~T}$ : visual and quantitative comparisons with $b=1000 \mathrm{~s} / \mathrm{mm} 2$. AJNR Am J Neuroradiol. 2008; 29(3):458-63..

[42] Soares JM, Marques P, Alves V, Sousa N. A hitchhiker's guide to diffusion tensor imaging, Front Neurosci. 2013, 7(31), doi: 10.3389/fnins.2013.00031.

[43] Le Bihan D, Mangin JF, Poupon C, Clark CA, Pappata S, Molko N, Chabriat H. Diffusion tensor imaging: concepts and applications, Journal of Magnetic Resonance Imaging 2001; 13(4): 534-546, doi: 10.1002/jmri.1076.

[44] Kingsley PB. Introduction to diffusion tensor imaging mathematics: Part II. Anisotropy, diffusion-weighting factors, and gradient encoding schemes, Concepts in Magnetic Resonance Part A 2006; 28 A(2): 123-154, doi: 10.1002/cmr.a.20049.

[45] Brodmann K. Brodmann's Localisation in the Cerebral Cortex - The Principles of Comparative Localisation in the Cerebral Cortex Based on Cytoarchitectonics, Springer 2006, ISBN-13: 978-0387-26917-7.

[46] Ern A, Guermond JL. Theory and practice of finite elements. Springer Science \& Business Media 2013.

[47] Saad Y. Iterative methods for sparse linear systems (2nd edt.), 2003, ISBN: 978-0-89871-534-7, doi: 10.1137/1.9780898718003.

[48] Cruz NF, Adachi K, Dienel GA. Rapid Efflux of Lactate From Cerebral Cortex During $\mathrm{K}^{+}$-Induced Spreading Cortical Depression. Journal of Cerebral Blood Flow and Metabolism. 1999 Apr 1;19(4):380-92.

[49] Gerardo-Giorda L, Kroos JM. A computational multiscale model of cortical spreading depression propagation, 2017, http://hdl.handle.net/20.500.11824/336, (submitted). 